\title{
Neuron-Specific FMRP Roles in Experience-Dependent Remodeling of Olfactory Brain Innervation during an Early-Life Critical Period
}

\author{
${ }^{\circledR}$ Randall M. Golovin, ${ }^{1}$ Jacob Vest, ${ }^{2}$ and ${ }^{\circledR}$ Kendal Broadie ${ }^{1,2,3,4}$ \\ ${ }^{1}$ Vanderbilt Brain Institute, ${ }^{2}$ Department of Biological Sciences, ${ }^{3}$ Department of Cell and Developmental Biology, and ${ }^{4}$ Department of \\ Pharmacology, Vanderbilt University and Medical Center, Vanderbilt University, Nashville, 37235, Tennessee
}

Critical periods are developmental windows during which neural circuits effectively adapt to the new sensory environment. Animal models of fragile X syndrome (FXS), a common monogenic autism spectrum disorder (ASD), exhibit profound impairments of sensory experience-driven critical periods. However, it is not known whether the causative fragile $\mathrm{X}$ mental retardation protein (FMRP) acts uniformly across neurons, or instead manifests neuron-specific functions. Here, we use the genetically-tractable Drosophila brain antennal lobe $(\mathrm{AL})$ olfactory circuit of both sexes to investigate neuron-specific FMRP roles in the odorant experience-dependent remodeling of the olfactory sensory neuron (OSN) innervation during an early-life critical period. We find targeted OSN class-specific FMRP RNAi impairs innervation remodeling within AL synaptic glomeruli, whereas global $d f m r 1$ null mutants display relatively normal odorant-driven refinement. We find both OSN cell autonomous and cell non-autonomous FMRP functions mediate odorant experience-dependent remodeling, with AL circuit FMRP imbalance causing defects in overall glomerulus innervation refinement. We find OSN class-specific FMRP levels bidirectionally regulate critical period remodeling, with odorant experience selectively controlling OSN synaptic terminals in AL glomeruli. We find OSN class-specific FMRP loss impairs critical period remodeling by disrupting responses to lateral modulation from other odorant-responsive OSNs mediating overall AL gain control. We find that silencing glutamatergic AL interneurons reduces OSN remodeling, while conversely, interfering with the $O S N$ class-specific $G_{A B A}$ signaling enhances remodeling. These findings reveal control of OSN synaptic remodeling by FMRP with neuron-specific circuit functions, and indicate how neural circuitry can compensate for global FMRP loss to reinstate normal critical period brain circuit remodeling.

Key words: critical period; Drosophila; fragile X mental retardation protein; fragile X syndrome; sensory experience; synapse elimination

\section{Significance Statement}

Fragile X syndrome (FXS), the leading monogenic cause of intellectual disability and autism spectrum disorder (ASD), manifests severe neurodevelopmental delays. Likewise, FXS disease models display disrupted neurodevelopmental critical periods. In the well-mapped Drosophila olfactory circuit model, perturbing the causative fragile $\mathrm{X}$ mental retardation protein (FMRP) within a single olfactory sensory neuron (OSN) class impairs odorant-dependent remodeling during an early-life critical period. Importantly, this impairment requires activation of other OSNs, and the olfactory circuit can compensate when FMRP is removed from all OSNs. Understanding the neuron-specific FMRP requirements within a developing neural circuit, as well as the FMRP loss compensation mechanisms, should help us engineer FXS treatments. This work suggests FXS treatments could use homeostatic mechanisms to alleviate circuit-level deficits.

Received Aug. 18, 2020; revised Dec. 4, 2020; accepted Dec. 8, 2020.

Author contributions: R.M.G., J.V., and K.B. designed research; R.M.G. and J.V. performed research; R.M.G. and J.V. analyzed data; R.M.G., J.V., and K.B. wrote the paper.

This work was supported by the National Institutes of Health Grant MH084989 (to K.B.). We thank Francois Bolduc for UAS-dfmr1 RNAi lines (1-1-7, 2-1; University of Alberta, Canada), Ron Davis for UAS-Rdl RNAi (8-10); The Scripps Research Institute Florida), llona Grunwald-Kadow for Or42a-mCD8:GFP (Technical University of Munich, Germany), Craig Montell for GtACR1-eYFP (University of California Santa Barbara), Mani Ramaswami for NP1227(LN1)-Gal4 (Trinity College
Dublin, Ireland), and Bloomington Drosophila Stock Center (Indiana University) for many lines. We also thank Dominic Vita, Danielle Kopke, and Jim Sears for their input. The authors declare no competing financial interests.

Correspondence should be addressed to Kendal Broadie at kendal.broadie@vanderbilt.edu. https://doi.org/10.1523/JNEUROSCI.2167-20.2020

Copyright $\odot 2021$ the authors 


\section{Introduction}

Critical periods are time windows when brain circuitry is particularly susceptible to initial sensory input driving activity-dependent remodeling (Hubel and Wiesel, 1970; Hensch, 2005). This refinement is impaired in a range of heritable neurologic disorders (Dölen et al., 2007; Greenhill et al., 2015; Krishnan et al., 2015; Meredith, 2015). Fragile X syndrome (FXS) patients exhibit profound developmental delays (Bailey et al., 1998; Roberts et al., 2016), and FXS disease models display disrupted critical periods (Dölen et al., 2007; Contractor et al., 2015). This leading monogenic cause of both intellectual disability and autism spectrum disorder is characterized by hypersensitivity to sensory stimuli and childhood activity-dependent seizures (Crawford et al., 2001; Hersh et al., 2011; Contractor et al., 2015). The causal fragile X mental retardation protein (FMRP) regulates activitydependent protein synthesis enabling experience-dependent synaptic plasticity (Brown et al., 2001; Darnell et al., 2001; Zalfa et al., 2003; Dölen et al., 2007), especially during critical periods (Bureau et al., 2008; He et al., 2014; Doll et al., 2017). Building evidence suggests specific FMRP roles in different brain circuits and cell types (Dahlhaus, 2018). Neuron class-specific FMRP genetic manipulations reveal striking differences controlling activity-dependent connectivity remodeling (Doll and Broadie, 2015), channel-binding (Brandalise et al., 2020), and translational control (Sawicka et al., 2019). Thus, it is crucial to test FMRP functions within specific neurons of defined brain circuits, and particularly FMRP roles during activity-dependent neural circuit remodeling in early sensory critical periods.

The Drosophila brain antennal lobe (AL) olfactory circuit provides an excellent model to study odorant sensory experience-dependent critical period remodeling (Devaud et al., 2003). Numerous studies have shown that exposing young animals to selected odorants changes $\mathrm{AL}$ circuit structure and function (Devaud et al., 2003; Sachse et al., 2007; Doll and Broadie, 2015; Golovin et al., 2019; Chodankar et al., 2020). Early work established that critical period odor exposure alters olfactory sensory neuron (OSN) connectivity in activated AL regions (Devaud et al., 2003; Sachse et al., 2007). Subsequent work showed that odorant exposure or optogenetic activity stimulation during just the first day following eclosion drives FMRP-dependent PN structural and functional remodeling (Doll and Broadie, 2015, 2016). Recently, we discovered a new form of experience-dependent OSN remodeling (Golovin et al., 2019), which reduces Or42a-expressing OSN innervation of a specific AL synaptic glomerulus following ethyl butyrate (EB) odorant exposure during the first 2 days of life, but not a week later. Furthermore, this remodeling requires functional odorant receptors, but not OSN output, and is reversed following prolonged removal from the odorant (Golovin et al., 2019). Together, these studies demonstrate that temporally restricted critical period odor experience refines AL glomeruli innervation and activity-dependent function. However, it remains unclear how reversible critical period OSN remodeling employs FMRP, and whether neuron-specific FMRP actions operate in AL circuit mechanisms.

Based on the key roles of FMRP in regulating critical period remodeling within the AL, we hypothesized an FMRP requirement in OSNs. To test this hypothesis, we assayed Or42a OSN innervation of the VM7 glomerulus following EB exposure during the well-mapped critical period (Golovin et al., 2019). Surprisingly, we find FMRP null mutants manifest normal OSN innervation refinement following EB exposure, whereas Or42atargeted FMRP RNAi strongly attenuates experience-dependent remodeling. Consistently, global FMRP RNAi mimics null mutants by not affecting synaptic remodeling, whereas Or42atargeted FMRP overexpression (OE) strongly enhances remodeling following EB exposure. These results indicate that balanced FMRP levels across EB-activated OSNs are required to tune LN input. We find that Or42a-targeted FMRP RNAi does not affect OSN remodeling after Or42a-specific optogenetic activation, but still attenuates circuit remodeling when Or42a OSN synaptic output is blocked. Importantly, we find that blocking glutamatergic AL interneuron neurotransmission as well as $\mathrm{GABA}_{\mathrm{A}} \mathrm{R}$ signaling disrupts Or42a OSN remodeling, suggesting that imbalanced FMRP levels perturb LN to Or42a OSN activity. Together, these results reveal neuronspecific FMRP functions in AL circuit critical period remodeling, and demonstrate that this circuit can restore normal function in the absence of FMRP.

\section{Materials and Methods}

\section{Drosophila genetics}

All animals were reared at $25^{\circ} \mathrm{C}$ before odor/light exposure. Animals were reared on a 12/12 h light/dark cycle except for light exposure experiments, for which animals were kept in darkness. All animals were fed on the standard Drosophila cornmeal molasses food. Initial experiments used animals of both sexes, but males show reduced critical period OSN innervation remodeling compared with females. Therefore, later experiments were conducted using only females to control the remodeling variability, and ensure a robust response to the odorant-dependent critical period OSN remodeling across the many experimental genotypes. All genotypes were confirmed with visible markers and/or PCR. Transgenic controls include $w^{-}$; UASmCD8::GFP/+; Or42a-Gal4/+, w-; Or42a-mCD8::4xGFP/Or42amCD8::4xGFP, $w$; Or42a-mCD8::4xGFP/+; Or42a-mCD8::4xGFP/+ and $w$; Or42a-mCD8::4xGFP/Or42a-mCD8::4xGFP. The genetic lines used for each figure are listed in Table 1.

\section{Odorant exposure}

Critical period odorant exposure was done as we previously reported (Golovin et al., 2019). Briefly; Animals were staged as dark pupa ( $4 \mathrm{~d}$ after puparium formation at $25^{\circ} \mathrm{C}$ ), separated based on both the sex and genotype. Fine wire mesh caps were secured onto the animal vials to allow good airflow, and the vials were then placed within larger airtight containers (3700 ml, Glasslock). In 1.5-ml microcentrifuge tubes, $1 \mathrm{ml}$ of mineral oil (Sigma-Aldrich) was placed alone (vehicle control), or with $10 \%, 15 \%, 20 \%$, or $25 \%$ EB (\% v/v in mineral oil; Sigma-Aldrich). Containers were placed in humidified $23^{\circ} \mathrm{C}$ incubators with a $12 / 12 \mathrm{~h}$ light/dark cycle. After $24 \mathrm{~h}$, the adult animals were rapidly transferred to new vials in clean chambers with a fresh odorant supply. Animals were then kept in the odorant chambers for another $24 \mathrm{~h}$. The entire odorant exposure period was $2 \mathrm{~d} ; 0-2 \mathrm{~d}$ posteclosion (dpe).

\section{Light exposure}

The optogenetic light exposure matched the above critical period odorant exposure paradigm. Dark-reared animals were staged as dark pupa, separated based on sex and genotype, and then transferred to a Petri dish $(35 \times 10 \mathrm{~mm}$ Falcon) with $3 \mathrm{ml}$ of food. The Petri dish was then placed in the same containers used above $(3700 \mathrm{ml}$, Glasslock) in dark, humidified $23^{\circ} \mathrm{C}$ incubators. Light was supplied through a custom-built cyan LED array $(515 \mathrm{~nm})$ controlled by an Arduino Uno (Arduino) using a custom script. The light exposure was $5-\mathrm{Hz} 50-\mathrm{ms}$ pulses $\left(337 \mu \mathrm{W} / \mathrm{mm}^{2}\right)$. After $24 \mathrm{~h}$, animals were rapidly transferred to a new dish in a clean chamber. The entire adult animal light exposure period was $2 \mathrm{~d}$; 0-2 dpe.

\section{Confocal imaging}

Staged animals were anesthetized on ice for at least 1-2 min, and then brains were dissected using fine forceps (Dumont \#5) in physiological saline (128 Mм NaCl, 2 мм KCl, 4 мM $\mathrm{MgCl}_{2}, 1.8 \mathrm{Mm} \mathrm{CaCl} 2,64.6$ мM sucrose, and 5 MM HEPES, pH 7.2; Sigma-Aldrich). Dissected brains were fixed for $30 \mathrm{~min}$ at room temperature (RT) in 4\% PFA (EMS)/4\% 
Table 1. List of experimental genotypes

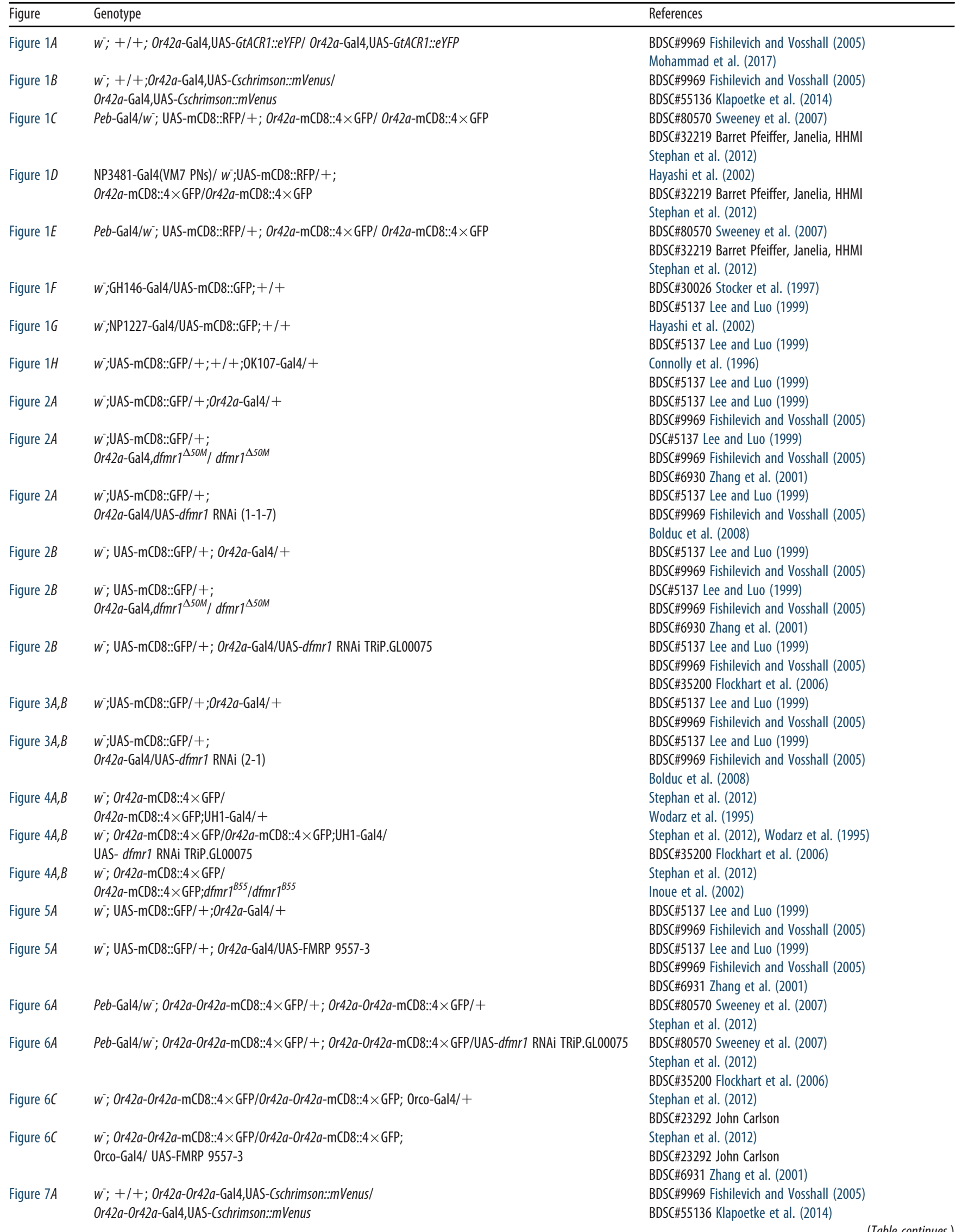


Table 1. Continued

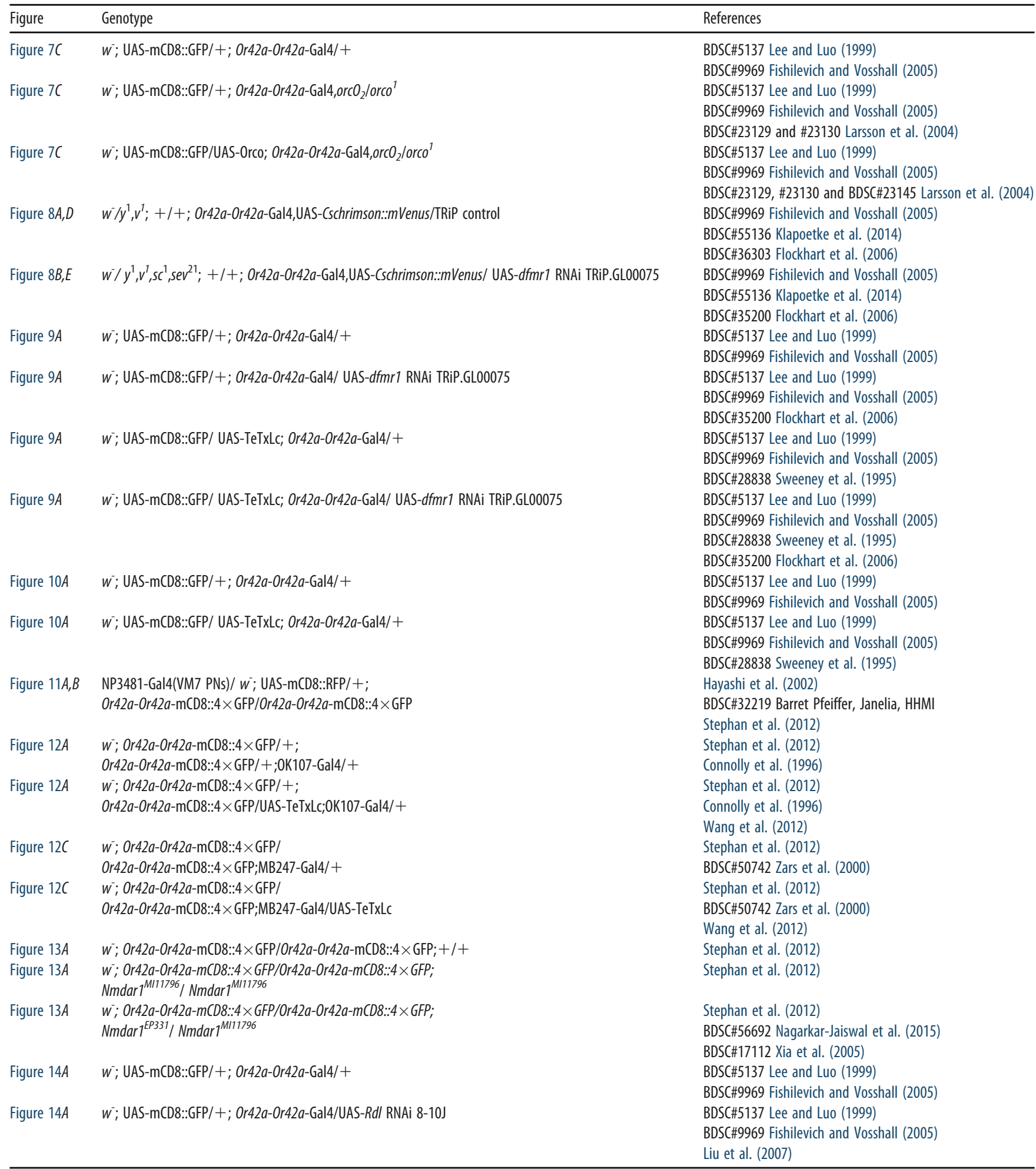

sucrose in PBS, pH 7.4 (Invitrogen). Brains were washed $3 \times$ with PBS and then blocked for $1 \mathrm{~h}$ in $1 \%$ BSA (Sigma-Aldrich) in PBS-T $(0.2 \%$ Triton X-100 in PBS; Fisher Chemical). Brains were then incubated with primary antibodies diluted in $0.2 \% \mathrm{BSA}$ in PBS-T at $4^{\circ} \mathrm{C}$ overnight $(\mathrm{O} /$ $\mathrm{N}$ ). Primary antibodies used were as follows: rabbit anti-GFP (Abcam 290; 1:3000), rat anti-RFP (Chromotek 5F8; 1:500), mouse anti-dFMRP (Abcam a10299 [6A15]; 1:125), and mouse anti-Bruchpilot [BRP; Developmental Studies Hybridoma Bank (DSHB), nc82; 1:50]. Brains were washed $3 \times$ for $20 \mathrm{~min}$ with $\mathrm{PBS}-\mathrm{T}$ and then incubated $\mathrm{O} / \mathrm{N}$ with secondary antibodies. Secondary antibodies used were as follows: Alexa
Fluor 488 goat anti-rabbit, Alexa Fluor 555 donkey anti-mouse, and Alexa Fluor 546 goat anti-rat (all at 1:250). Brains were then washed in PBS-T $3 \times$ for $20 \mathrm{~min}$, followed by PBS $1 \times$ for $20 \mathrm{~min}$. Brains were then rinsed with $\mathrm{dH}_{2} \mathrm{O}$ and mounted in Fluoromount (EMS 17984-25) on a glass slide (ProbeOn Microscope Slides, Fisherbrand) with a glass coverslip (No. 1.5H, Carl Zeiss). Double-sided adhesive tape (Scotch) was used to raise the coverslips above the brains, and clear nail polish (Sally Hansen) was used to seal the coverslip to the slide. For maxillary palp studies, whole proboscises were dissected and processed as above, except with longer fixation (45 min), longer primary/secondary antibody 
incubations (38-42 h) and no double-sided tap used for mounting. The whole head image (see Fig. 1) was taken using an iPhone 6 (Apple) through the oculars of a Leica dissecting scope with both white light and filtered mercury lamp light for illumination. Confocal images were collected on a 510 META laser-scanning confocal microscope (Carl Zeiss) with $40 \times$ and $63 \times$ oil-immersion objectives. Images taken with the $40 \times$ lens were collected at $1024 \times 1024$ resolution with a $Z$ slice of $1-\mu \mathrm{m}$ thickness. Images taken with the $63 \times$ lens were collected at $2048 \times 2048$ resolution with a $Z$ slice thickness of $0.8 \mu \mathrm{m}$. The microscope and imaging settings were kept constant within every experiment.

\section{Quantification}

$\mathrm{AL}$ glomeruli measurements were done as previously reported (Golovin et al., 2019). Briefly, blinded brain images were visualized in Image (NIH) with the quantification channel isolated. A maximum intensity projection that captured the whole VM7 glomerulus was used to generate a region of interest (RoI). This RoI was used with the FIJI plugin 3D Object Counter (Schindelin et al., 2012) to quantify the RoI volume. For every experiment, the threshold was set at a constant value that minimized the noise. In controls, the $3 \mathrm{D}$ Object Counter output typically contained two RoIs, which were summed. In experimental conditions, when the glomerular RoI was more discontinuous, all the regions were summed. To control for variations of signal across the different labeling constructs, antibody aliquots and experimental days, glomerulus volumes were normalized to the control mean for each experimental replicate. For the maxillary palp, blinded Z-stack images were analyzed using ImageJ. A maximum intensity projection was used to capture all the Or42a OSNs. For the fluorescence intensity measurements, one RoI for each Or42a-positive soma was generated using the GFP signal. ImageJ was then used to quantify the mean intensity for each soma in both the GFP and FMRP channels.

\section{Statistics}

All tests were done using Prism 8 (GraphPad). For comparisons with more than or equal to two genotypes, a two-way ANOVA was used with odorant/light and genotype as independent variables. Follow-up pairwise comparisons were done using $t$ tests, with Sidak's multiple comparisons correction. Interactions between genotype and odorant/light exposure were tested with the two-way ANOVA interaction term. A ROUT outlier test was done for data with Q set to $1 \%$. Some genotypes showed altered basal glomerulus innervation volumes in the vehicle-exposed control animals. While the ANOVA post hoc test can distinguish whether the EB-treated means are different, it does not account for differences in glomerulus volume of vehicle-treated controls. With only two independent variables, an ANOVA $(2 \times 2)$ interaction term can be attributed to the specific genotype/odorant exposure. However, if more than the independent variables occur, the interaction term cannot be used for pairwise comparisons between genotypes. Therefore, in all the cases with more than two independent variables, a linear regression with $t$ tests comparing pairwise interaction terms was used to assess EB treatment effects while controlling for any changes in the basal glomerulus innervation volume. Regression coefficients for each genotype interaction term represent the differences in the treatment effect on the experimental genotype compared with the control genotype. If the interaction term of an experimental genotype were zero, this would indicate the effect of treatment had not changed in the genotype compared with the matched control (glomerular innervation
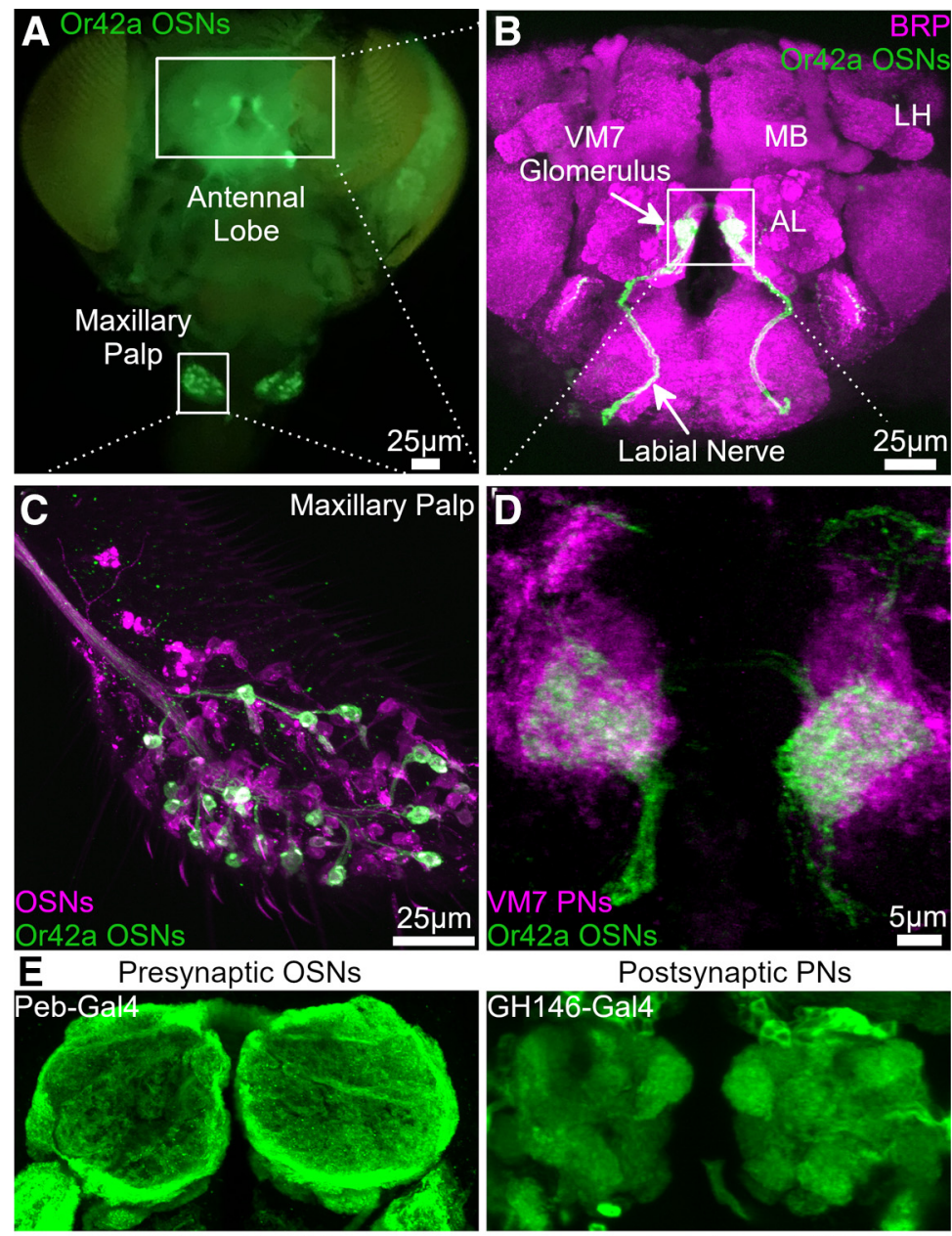

GABAergic LNs
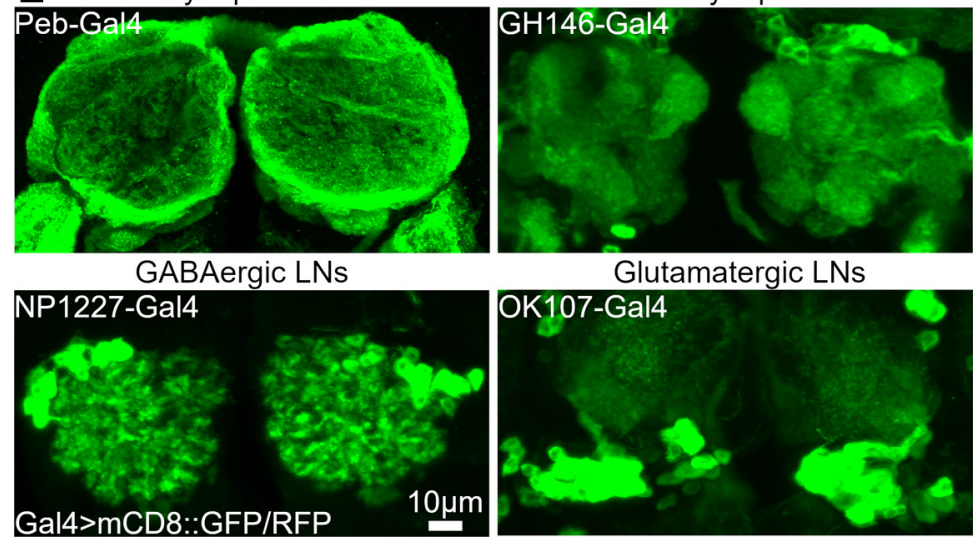

Glutamatergic LNs

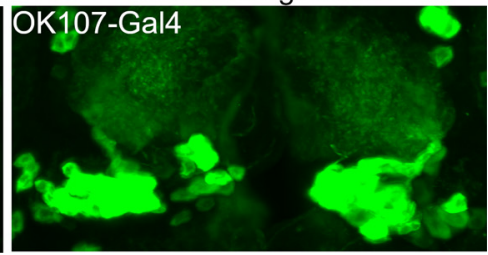

Figure 1. Maxillary palp (MP) to antennal lobe (AL) olfactory circuitry and neuron class-specific drivers $A$, Whole Drosophila head showing Or42a olfactory sensory neuron (OSN) innervation pattern (0r42aGal4>UAS-GtACR1-eYFP; green). OSN cell bodies in the maxillary palp (MP) project to the antennal lobe (AL). B, Or42a OSN innervation (Or42a-Gal4>UAS-CsChrimson::mVenus; green) of central brain (larger box in A) co-labeled for presynaptic Bruchpilot (BRP; magenta). Or42a OSNs extend axons via the labial nerve (bottom arrow) to terminate in the VM7 glomeruli (top arrow) of each AL. AL glomeruli postsynaptic projection neurons (PNs) send axons to the mushroom body (MB) and lateral horn (LH) in each brain hemisphere. C, Or42a OSN cell bodies (Or42a-mCD8-4xGFP; green) in MP (smaller box in $(\boldsymbol{A})$ co-labeled for all OSN somata (Pebbled (Peb)-Gal4>UAS-mCD8::RFP; magenta). D, Or42a OSN axonal termini (0r42a-mCD8-4xGFP; green) and postsynaptic PNs (NP3481-Gal4>UAS-mCD8::RFP; magenta) in the AL VM7 (white box in $(\boldsymbol{B})$. $\boldsymbol{E}$, Neuron class-specific Gal4 drivers expressing UAS-mCD8::GFP in presynaptic OSNs (Peb-Gal4; top left), postsynaptic PNs (GH146-Gal4; top right), GABAergic local interneurons (LNs, NP1227-Gal4; bottom left) and Glutamatergic LNs (OK107-Gal4; bottom right) of the brain AL.

changed by the same amount). If the interaction term were significantly greater than zero, this would indicate that the treatment had not reduced innervation as much in the experimental condition as in control. If the interaction term were significantly less than zero, this would indicate that the treatment had reduced the innervation by more in the experimental condition compared with the control. To display regression analyses, bar values of each genotype show the difference between vehicle and treatment conditions. Error bars are the sum of the error of the EB effect regression plus the error of the genotype effect regression. The sample size $(n)$ is the number of brains or maxillary 
A

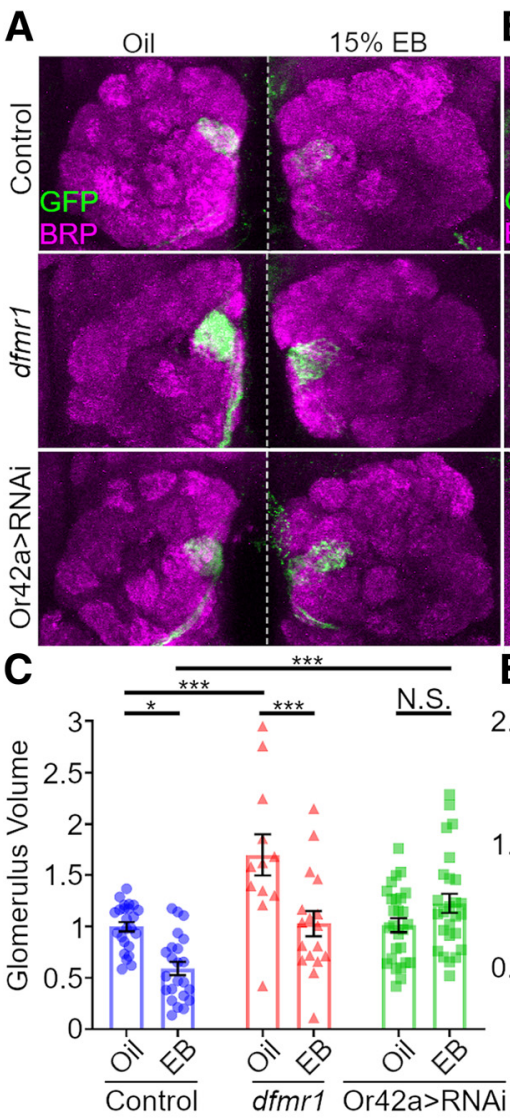

B
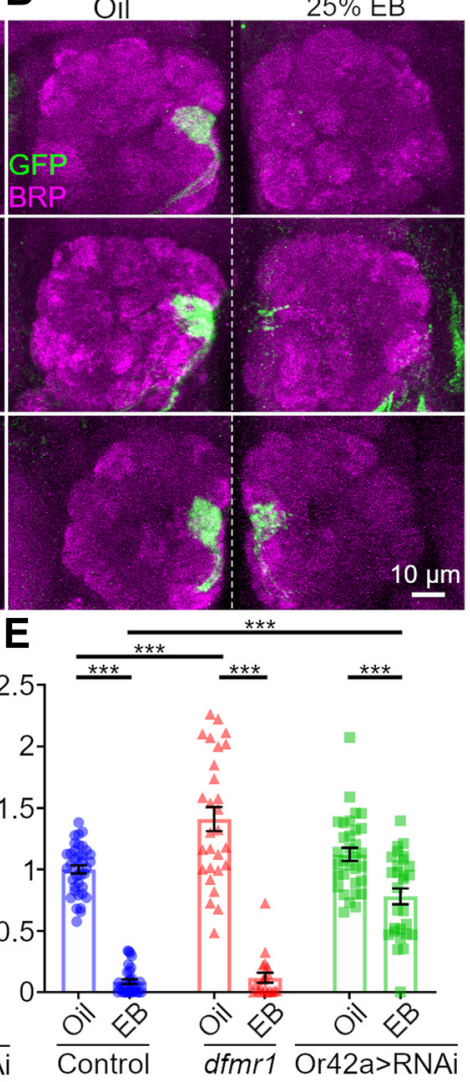

D

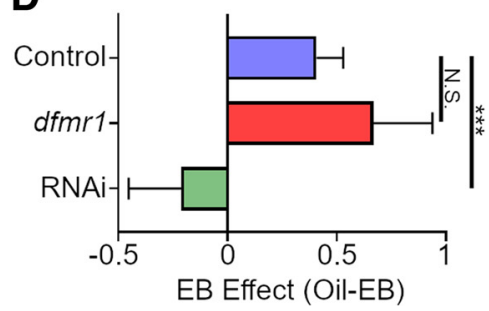

$\mathbf{F}$

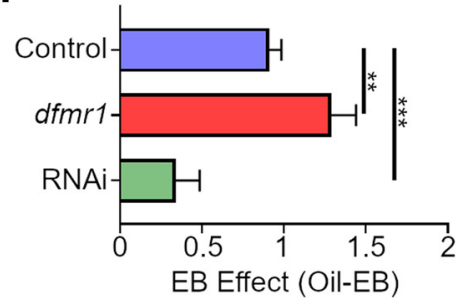

Figure 2. Or42a OSN-specific FMRP loss impairs VM7 innervation critical period remodeling $\boldsymbol{A}, \boldsymbol{B}$, Representative confocal maximum intensity projections of AL innervation by 0r42a 0SNs (Or42a-Gal4>UAS-mCD8::GFP; green) co-labeled for presynaptic BRP (magenta). Exposure to mineral oil vehicle (oil; left) or odorant (EB) during the critical period (0-2 dpe) at either $(\boldsymbol{A})$ 15\% EB or $(\boldsymbol{B})$ 25\% EB (\%V/V). Three genotypes are shown: Or42a-Gal4>mCD8::GFP transgenic control (top), dfmr1 null (dfmrr ${ }^{50 M}$; middle), and Or42a-Gal4 targeted dfmr1 RNAi (A, 1-1-7; $\boldsymbol{B}$, TriP GL00075). C, Quantification of Or42a-OSN AL VM7 glomerulus innervation volumes comparing oil vehicle and $15 \% \mathrm{~EB}$, normalized to vehicle control. $\boldsymbol{D}$, The difference between oil and EB exposure for each genotype. $\boldsymbol{E}$, Quantification of the Or42a-0SNs VM7 innervation at $25 \%$ EB for all three genotypes. $\boldsymbol{F}$, The difference between oil and EB exposure for each genotype. The scatter plots show all data points and the mean \pm SEM for each assay. The bar graphs show mean \pm SER for each assay. The significance is indicated as not significant (N.S.; $p>0.05$ ), or significant at $* p<0.05, * * p<0.01, * * * p<0.001$.

palps. When comparing data with only two conditions, Sidak's corrected $t$-tests were employed. Line graphs are of linear regression analyses. ANOVAs are displayed as scatter plots with mean \pm SEM. The EB effects from the linear regression analyses are displayed as bar graphs with mean \pm standard error of the regression (SER). Significance is shown as not significant (N.S.; $p>0.05$ ) or significant at $* p<0.05, * * p<0.01, * * * p<0.001$.

\section{Results}

OSN-specific FMRP loss disrupts odor experience critical period remodeling of $\mathrm{AL}$ innervation

The well-mapped and genetically tractable neuron classes of the Drosophila AL olfactory circuit make it a very powerful system to study the mechanisms of sensory experience-dependent remodeling. The AL is the first brain synaptic node of the olfactory circuit (Fig. 1A,B; Wilson, 2013). Odorants transduced by OSNs in both the antenna and maxillary palps (Fig. $1 C$ ) project axons along antennal and labial nerves (Fig. 1B, bottom arrow), respectively, to innervate OSN-specific AL synaptic glomeruli (Fig. $1 D, E$, top left; Wilson, 2013). Each OSN generally expresses a single olfactory receptor (e.g., Or42a; Fig. $1 A-D$ ), in addition to the pan-OSN Orco co-receptor (Larsson et al., 2004; Couto et al., 2005). Each AL glomerulus receives convergent OSNs that express only that single olfactory receptor (Fig. $1 B$, boxed region, $D$ ). Within each $\mathrm{AL}$ glomerulus, OSNs synapse onto projection neurons (PNs; Fig. 1D,E, top right). PNs subsequently send olfactory information to the central brain mushroom body (MB) and lateral horn (Fig. 1B; Jefferis et al., 2002; Marin et al., 2002). Within the AL, multiple local interneurons (LNs) synapse broadly to provide modulation of OSN-PN excitatory (cholinergic) connections. The LNs release a variety of neuromodulators that can both inhibit and excite this olfactory neurotransmission, including GABA (Fig. 1E, bottom left) and glutamate (Fig. $1 E$, bottom right; Chou et al., 2010; Wilson, 2013). In addition, LNs can also signal through gap junction mediated electrical synapses (Huang et al., 2010). OSN-AL synaptic connectivity is strongly altered by critical period olfactory experience, providing an ideal system to test circuit level FMRP roles in different neuron classes during synaptic remodeling.

We have reported that odorant exposure to EB during the critical period ( $0-2$ dpe) causes a large-scale reduction of Or42a OSN innervation of the AL VM7 glomerulus (Fig. 1A-D; Golovin et al., 2019). Based on previous work from our lab and others on the role of FMRP in mediating AL circuit remodeling (Sudhakaran et al., 2014; Doll and Broadie, 2015), and FMRP roles in regulating the critical period plasticity in other neural circuits (Dölen et al., 2007; Bureau et al., 2008; Gonçalves et al., 2013), we hypothesized that FMRP has a role in OSN remodeling. To test this idea, we assayed Or42a OSN innervation of the AL VM7 glomerulus following 0 - to 2-dpe critical period EB exposure in $d f m r 1$ null mutants $\left(d f m r 1^{50 \mathrm{M}}\right)$, Or42a OSN-targeted $d f m r 1$ RNAi, and matched genetic background controls. To visualize Or42a OSN innervation of the VM7 glomerulus, the membrane marker UAS-mCD8::GFP was driven with Or42a-Gal4 (Fig. 2). Innervation volume was assayed between animals exposed to the mineral oil vehicle compared with $\mathrm{EB}$ dissolved in the oil at lower concentrations (15\% EB; Fig. 2A) or higher concentrations ( $25 \%$ EB; Fig. $2 B$ ) during the 0 - to 2 -dpe critical period. The three genotypes compared were the transgenic control (Or42a- 
Gal4>mCD8::GFP; Fig. 2A,B, top panels), $d f m r 1$ null mutant (dfmrl ${ }^{50 \mathrm{M}}$; Fig. $2 A, B$, middle panels), and Or42a OSN-targeted $d f m r 1$ knock-down (Or42a-Gal4 $>d f m r 1$ RNAi; Fig. $2 A, B$, bottom panels). Sample images of Or42a OSN AL innervation, quantified VM7 glomerular innervation volumes, and quantified genotype effect comparisons are all shown in Figure 2.

Control animals exposed to EB during the 0- to 2-dpe critical period show striking reduction of Or42a OSN innervation of the VM7 glomerulus (Fig. $2 A, B$, top). In contrast to our working hypothesis, animals completely lacking FMRP (dfmr $1^{50 \mathrm{M}}$ null) exhibit a similar large reduction in VM7 innervation following critical period EB odorant exposure, despite an increase in the basal glomerulus innervation (Fig. $2 A, B$ middle). In stark contrast, Or42a OSN-targeted FMRP RNAi strongly suppresses the EB odorant-dependent loss of Or42a OSN innervation, supporting the hypothesis (Fig. $2 A, B$, bottom). An ANOVA $(3 \times 2)$ was used to compare the effects on the three genotypes exposed to vehicle control versus 15\% EB (Fig. 2C). Quantification of the Or42a OSN innervation volume shows significant effects of both genotype $\left(F_{(2,123)}=17.20, p=2.59 \mathrm{e}-7\right)$ and $\mathrm{EB}$ exposure $\left(F_{(1,123)}=14, p=0.0003\right)$, with a significant interaction between genotype and odorant exposure $\left(F_{(2,123)}=12.21, p=1.45 \mathrm{e}-5\right)$. The remodeling is EB concentration-dependent, as increasing the odorant concentration $(25 \% \mathrm{~EB})$ causes a larger innervation reduction (Fig. $2 A$ vs $B$ ). An ANOVA $(3 \times 2)$ comparing transgenic control, $d f m r 1$ null, and Or42a OSN-targeted FMRP RNAi shows significant effects of both genotype $\left(F_{(2,170)}=29.09\right.$, $p=1.363 \mathrm{e}-11)$ and $\mathrm{EB}$ exposure $\left(F_{(1,170)}=329.7, p=3.113 \mathrm{e}-59\right)$, with a significant interaction between the genotype and the odorant $\left(F_{(2,170)}=33.20, p=6.732 \mathrm{e}-13\right.$; Fig. $\left.2 E\right)$. Quantitative analyses with pairwise comparisons as well as linear regression models further indicate that Or42a OSN-specific FMRP loss suppresses critical period remodeling.

Unpaired $t$ tests with Sidak's corrections were done to compare the ANOVA conditions. In controls, VM7 innervation is significantly reduced following $15 \%$ EB critical period exposure [normalized vehicle control (oil) $1.0 \pm 0.046$ ( $n=24$ brains) vs $15 \%$ EB $0.592 \pm 0.065(n=23) ; t_{(123)}=3.331, p=0.017$; Fig. $2 C$, bottom left bar]. A stronger reduction (91.2\% vs $40.8 \%$ ) occurs with $25 \% \mathrm{~EB}$ [oil control $1.0 \pm 0.033$ ( $n=37$ brains) vs $25 \% \mathrm{~EB}$ $0.088 \pm 0.019(n=32) ; t_{(170)}=12.47, p=1.38 \mathrm{e}-24$; Fig. $2 E$, bottom left]. Compared with vehicle control animals, $d f m r 1$ nulls have significantly greater VM7 basal innervation in both $15 \%$ EB [oil $d f m r 1$ null $1.698 \pm 0.2(n=12) ; t_{(123)}=4.705, p=0.0001$; Fig. 2C, middle bar] and 25\% EB experiments [oil $d f m r 1$ null $1.41 \pm 0.098(n=28) ; t_{(170)}=5.41, p=3.18 \mathrm{e}-6$; Fig. $2 E$, middle bar]. Nevertheless, there is still a strong reduction in innervation following critical period EB odorant exposure (Fig. 2A, $B$, middle). Following 15\% EB, null mutants show a significant loss of innervation volume [oil $d f m r 1$ null vs $15 \% \mathrm{~EB} d f m r 1$ null $1.03 \pm 0.12(n=17) ; t_{(123)}=4.224, p=0.0007$; Fig. $2 C$, bottom center bar]. Likewise, null $d f m r 1$ VM7 glomerular innervation is significantly reduced following $25 \%$ EB exposure compared with the oil-exposed controls [25\% EB dfmr1 null $0.12 \pm 0.041(n=19) ; t_{(170)}=14.33, p=7.12 \mathrm{e}-30$; Fig. $2 E$, bottom center]. Similar to control animals, the effect of the higher $25 \% \mathrm{~EB}$ exposure is greater than the lower $15 \% \mathrm{~EB}$ exposure in the $d f m r 1$ null mutants $(91.5 \%$ vs $39.3 \%)$. These results indicate FMRP loss has an experience-independent function regulating basal glomerulus innervation, but little impact on experiencedependent remodeling.
In direct contrast to $d f m r 1$ nulls, FMRP RNAi targeted only to Or42a OSNs using two different constructs (1-1-7, Bolduc et al., 2008; TRiP GL00075, Flockhart et al., 2006; Greenblatt and Spradling, 2018) strongly impairs critical period remodeling (Fig. $2 A, B$, bottom). Innervation volume in vehicle knockdown animals is not significantly different from controls $[15 \%$ EB: oil Or42a OSN-targeted FMRP RNAi $1.01 \pm 0.069(n=27)$; $t_{(123)}=0.1197, p>0.9999 ; 25 \%$ EB: oil Or42a OSN-targeted FMRP RNAi $\left.1.12 \pm 0.053(n=32) ; t_{(170)}=1.663, p=0.79\right]$. Moreover, $15 \%$ EB critical period exposure does not significantly alter VM7 innervation [15\% EB $1.23 \pm 0.09 \quad(n=26)$; $t_{(123)}=1.841, p=0.6527$; Fig. $2 C$, bottom right bar]. At $25 \% \mathrm{~EB}$, innervation remodeling is also suppressed with Or42a OSN-targeted FMRP knock-down compared with vehicle controls [25\% EB $0.78 \pm 0.064(n=28) ; t_{(170)}=4.335, p=0.0004$; Fig. $2 E$, bottom right]. Since targeted FMRP removal in Or42a OSNs significantly reduces odorant experience-dependent remodeling of VM7 innervation, EB exposure in the knock-down condition was directly compared with the EB control to show a significantly reduced effect on VM7 innervation, at both $15 \%$ and $25 \%$ EB concentrations [15\% EB control vs $15 \%$ EB Or42a OSN-targeted FMRP RNAi; $t_{(123)}=5.279, p=8.52 \mathrm{e}-6$ (Fig. $2 C$, top bar); $25 \%$ EB control vs $25 \%$ EB Or42a OSN-targeted FMRP RNAi; $t_{(170)}=8.847, p=1.69 \mathrm{e}-14$ (Fig. $2 E$, top)]. The inability of FMRP RNAi to completely block OSN remodeling after $25 \%$ EB exposure could indicate that rather than impairing the mechanism directly it alters the OSN response to the odorant, thereby increasing the stimulus threshold needed for innervation loss. These results suggest that Or42a OSN-specific FMRP removal within the AL circuit has a significant impact on odorant experience critical period remodeling, but required further analyses to account for variations in basal innervation volume.

Differences in basal OSN innervation between genotypes complicates comparing the EB-exposed conditions. Therefore, a linear regression model was used to analyze EB exposure compared with vehicle, while controlling for genotype differences (see Materials and Methods). Unpaired $t$ tests of the null hypothesis that genotype does not affect the treatment relationship were done compare regression coefficients for each interaction term. Regression analyses show $d f m r 1$ null interaction with $15 \% \mathrm{~EB}$ exposure is not significant $\left(d f m r 1 x 15 \%\right.$ EB $\beta=-0.26 \pm 0.2 ; t_{(123)}=1.302, p=0.1953$; Fig. $2 D$, top), indicating no significant impact from global FMRP loss on EB exposure effects. With $25 \% \mathrm{~EB}$, the $d f m r 1$ null regression coefficient becomes significant $(\beta=$ $-0.38 \pm 0.12 ; t_{(170)}=3.267, p=0.0013$; Fig. $2 F$, top), indicating FMRP removal augments innervation loss from odorant exposure. Consistent with the pairwise comparisons between the control genotype and Or42a OSN-targeted FMRP RNAi, regression analyses show significant interactions at both $15 \%$ and $25 \%$ EB (Or42a OSN-targeted FMRP RNAix15\% EB $\beta=0.62 \pm 0.17 ; t_{(123)}=3.687$, $p=0.0003$; Or42a OSN-targeted FMRP RNAix $25 \%$ EB $\beta=$ $0.57 \pm 0.11 ; t_{(170)}=5.334, p=4.54 \mathrm{e}-6$; Fig. $2 D, F$, bottom) Together, these results indicate that FMRP has two roles: (1) a cell non-autonomous role regulating basal Or42a OSN-VM7 innervation, with $d f m r 1$ nulls displaying experience-independent increased glomeruli innervation; and (2) an OSN cell autonomous role regulating critical period olfactory experience-dependent remodeling, with Or42a-specific FMRP removal, but not global FMRP loss, limiting innervation refinement. 


\section{Sex-specific differences in early odorant experience critical period remodeling of $A L$ innervation}

In experiments testing the role of FMRP in critical period OSN remodeling, we observed that much of the variation between animal responses to EB exposure could be attributed to sex. To quantify this sex difference, we compared male and female animals following exposure to vehicle control and $20 \%$ EB from 0 to 2 dpe. To further validate our finding that Or42a-targeted RNAi against FMRP disrupts EB-dependent AL innervation remodeling, we used a third RNAi targeting FMRP (2-1; Bolduc et al., 2008). After odorant exposure, the volume of the Or42a OSN innervation of the VM7 glomerulus was assessed by quantifying Or42a-Gal4>UAS-mCD8::GFP. Animals exposed to $\mathrm{EB}$ during the critical period show pronounced changes in VM7 glomeruli, with sparser innervation and the appearance of OSN puncta (Fig. $3 A, B$ ). In EB-exposed males, OSN puncta occur in both controls and $d f m r 1$ RNAi animals, but the exposure has relatively little impact on the glomerulus innervation volume (Fig. 3A). Or42a-targeted $d f m r 1$ RNAi males lack any VM7 innervation loss but produce more OSN puncta (Fig. $3 A$, bottom right). In EB-exposed females, there is a much more pronounced response to odorant experience during the critical period, with a strong shift toward greater innervation loss but with fewer OSN puncta (Fig. 3B). As with the males, females expressing Or42a-targeted $d f m r 1$ RNAi exhibit very altered critical period remodeling, with the appearance of greater numbers of OSN puncta and much less loss of the overall VM7 glomerulus innervation compared with control females (Fig. $3 B$, bottom right). Representative images for both the sexes and both the genotypes, together with the quantitative innervation measurements, are shown in Figure 3.

A three-way ANOVA $(2 \times 2 \times 2)$ comparing innervation volume reveals significant effects of EB exposure $\left(F_{(1,206)}=17.47\right.$, $p=4.313 \mathrm{e}-5)$, genotype $\left(F_{(1,206)}=21.80, p=5.45 \mathrm{e}-6\right)$ and sex $\left(F_{(1,206)}=57.26, p=1.251 \mathrm{e}-12\right.$; Fig. $\left.3 C\right)$. There are significant interactions between odorant and genotype $\left(F_{(1,206)}=26.87\right.$, $p=5.179 \mathrm{e}-7)$ as well as sex $\left(F_{(1,206)}=63.83, p=9.485 \mathrm{e}-14\right)$, but not genotype and sex $\left(F_{(1,206)}=0.005, p=0.9427\right)$. EB-exposed control males show only a small loss in innervation volume [oil $1 \pm 0.036(n=26)$ vs EB $0.91 \pm 0.097(n=25)$; multiple comparisons with Sidak's correction, $t_{(206)}=0.9129, p>0.9999$; Fig. $3 C$, bottom left bar] compared with $d f m r 1$ RNAi males with significantly greater innervation relative to vehicle exposure [oil $0.955 \pm 0.1(n=26)$ vs EB $1.412 \pm 0.099(n=25) ; t_{(206)}=4.636$, $p=0.0002$; Fig. $3 C$, second bottom bar]. EB-exposed control females show a much greater loss of innervation [oil $1.0 \pm 0.037$ $(n=28)$ vs $\mathrm{EB} 0.187 \pm 0.029(n=28) ; t_{(206)}=8.634, p=4.55 \mathrm{e}-14$; Fig. $3 C$, third bottom bar], with Or $42 a-G a l 4>d f m r 1$ RNAi causing severely attenuated remodeling [RNAi oil $0.996 \pm 0.063$ $(n=28)$ vs $\mathrm{EB} 0.635 \pm 0.053(n=28) ; t_{(206)}=3.831, p=0.0047$; Fig. $3 C$, right bottom bar]. Quantification reveals that Or42a-targeted $d f m r 1$ RNAi causes significantly altered innervation in both sexes (control EB male vs RNAi EB Male; $t_{(206)}=5.042$, $p=2.81 \mathrm{e}-5$; control EB female vs RNAi EB female; $t_{(206)}=4.756$, $p=0.0001$; Fig. $3 C$, second bars). EB-exposed females have significantly less innervation than males in both controls and with $d f m r 1$ RNAi (control EB male vs control EB female; $t_{(206)}=$ 7.457, $p=6.78 \mathrm{e}-11$; RNAi EB male vs RNAi EB female; $t_{(206)}=$ $8.02, p=2.2 \mathrm{e}-12$; Fig. $3 C$, top two bars). Together, these results indicate critical period EB exposure causes a more robust loss of glomerulus innervation in control females, with Or42a-targeted $d f m r 1$ RNAi strongly attenuating this OSN remodeling. For

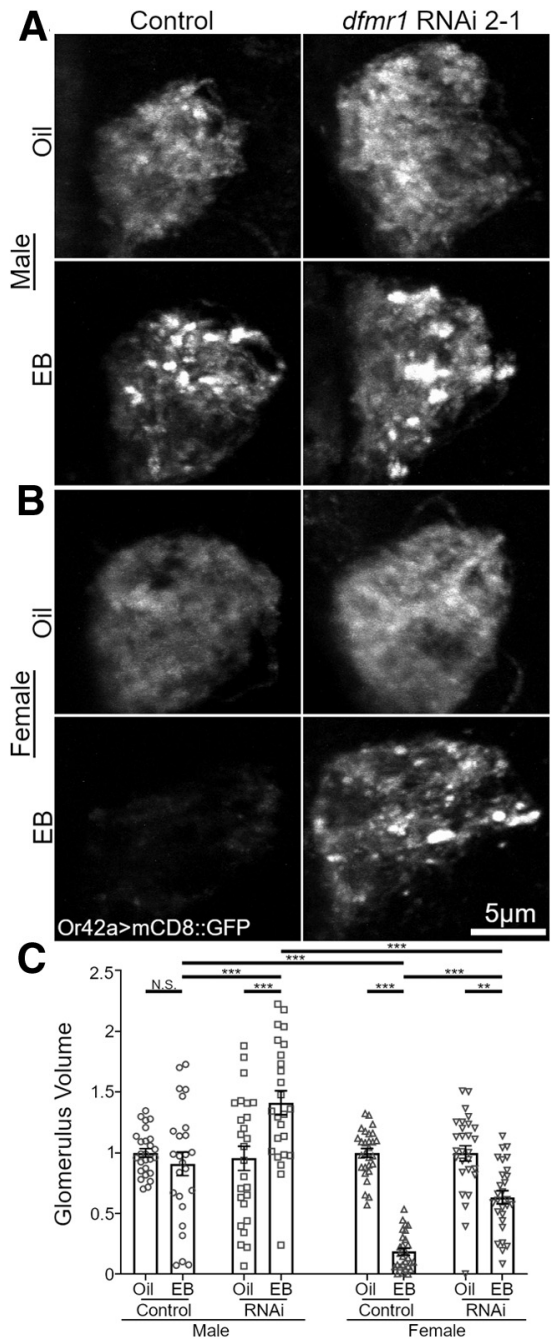

Figure 3. Sex-specific differences in EB-dependent critical period remodeling of $A L$ innervation. $\boldsymbol{A}$, Representative confocal maximum intensity projections of Or42a OSNs innervating the male AL VM7 glomerulus (Or42a-Gal4>UAS-mCD8::GFP; white). $\boldsymbol{B}$, Representative images from females under identical conditions. All animals were exposed to mineral oil vehicle (top), or 20\% EB odorant (bottom) during the 0- to 2-dpe critical period. The paired genotypes shown are the following: the transgenic control (Or42a-Gal4>mCD8::GFP; left column) and the same transgenic line with dfmr1 RNAi expression (Or42a-Gal4 $>d f m r 1$ RNAi 2-1; right column). C, Quantification of the Or42a OSN VM7 innervation volumes for both genotypes, treatment conditions, and sexes. Scatter plots show all data points and the mean \pm SEM. The significance is indicated as not significant (N.S.; $p>0.05$ ), or significant at $* * p<0.01$ and $* * * p<0.001$.

consistency and clarity in dissecting the causal mechanisms, females were employed in subsequent experiments.

\section{Null $d f m r 1$ mutants and global FMRP RNAi animals both maintain critical period remodeling}

Because of the above surprising difference between $d f m r 1$ null mutants and Or42a OSN-targeted $d f m r 1$ RNAi, we wanted to test the conclusion that neuron class-specific differences (Or42a OSN-targeted vs global) rather than technical differences (null mutant vs all three RNAi knock-downs) accounts for the result. We differentiated between these two possibilities by testing olfactory experience-dependent remodeling of Or42a OSN VM7 innervation with a global FMRP knockdown (UH1-Gal4>dfmr1 RNAi; Wodarz et al., 1995) and transgenic control lacking $d f m r 1$ RNAi, compared with a second $d f m r 1$ null mutant $\left(d f m r 1^{B 55}\right.$; Inoue et al., 2002). 
Animals of these three genotypes expressing a membrane-bound GFP under direct control of the Or42a promoter (Or42a-mCD8::4xGFP; Stephan et al., 2012) were exposed to either oil vehicle or $20 \%$ EB during the $0-2$ dpe critical period. If all three genotypes show the strong reduction in Or42a OSN VM7 innervation following EB exposure relative to the vehicle control, then the result supports neuron classspecific FMRP roles within the AL olfactory circuit. On the other hand, if the UH1-Gal4 $>d f m r 1$ RNAi animals show an impaired response to odorant experience during the critical period, then this would suggest an important difference between the FMRP null mutant and RNAi knock-down in mediating the olfactory experiencedependent remodeling phenotype. Representative images of brain FMRP levels and VM7 glomerulus innervation, as well as quantifications for all conditions, are shown in Figure 4.

In first testing our genetic tools, both global $d f m r 1$ RNAi and the homozygous $d f m r 1^{B 55}$ mutation led to an indistinguishable complete loss of brain FMRP expression compared with the robust FMRP levels in the matched transgenic controls (Fig. 4A, top vs middle and bottom). This agrees with previous reports on these lines (Inoue et al., 2002; Greenblatt and Spradling, 2018), showing a loss of detectable FMRP. As in the above experiments, exposing control animals to EB during the 0- to 2-dpe critical period causes a stark reduction of Or42a OSN VM7 glomerulus innervation (Fig. $4 B$, top). Importantly, the global UH1Gal4>dfmr1 RNAi animals look remarkably like the null mutants (Fig. $4 B$, middle). Global FMRP removal leads to an increase in the basal oil-exposed Or42a OSN innervation volume, while maintaining robust olfactory experience-dependent remodeling. As in the $d f m r 1^{50 M}$ null mutant above, the alternate $d f m r 1^{B 55}$ null mutant shows an indistinguishable response to both oil vehicle and EB exposure, with vehicle-treated animals showing a larger innervation volume compared with controls, but still maintaining the robust innervation loss following critical period EB odorant exposure (Fig. 4B, bottom). Note also the characteristic Or42a OSN puncta in the VM7 glomeruli of both the global dfmr 1 knock-down and null mutant following critical period EB exposure (Fig. 4B, middle and bottom arrows) suggesting a dynamic stage in the OSN remodeling process, as we reported previously (Golovin et al., 2019). Together, these results support an OSN-specific FMRP role in olfactory experience-dependent critical period remodeling.

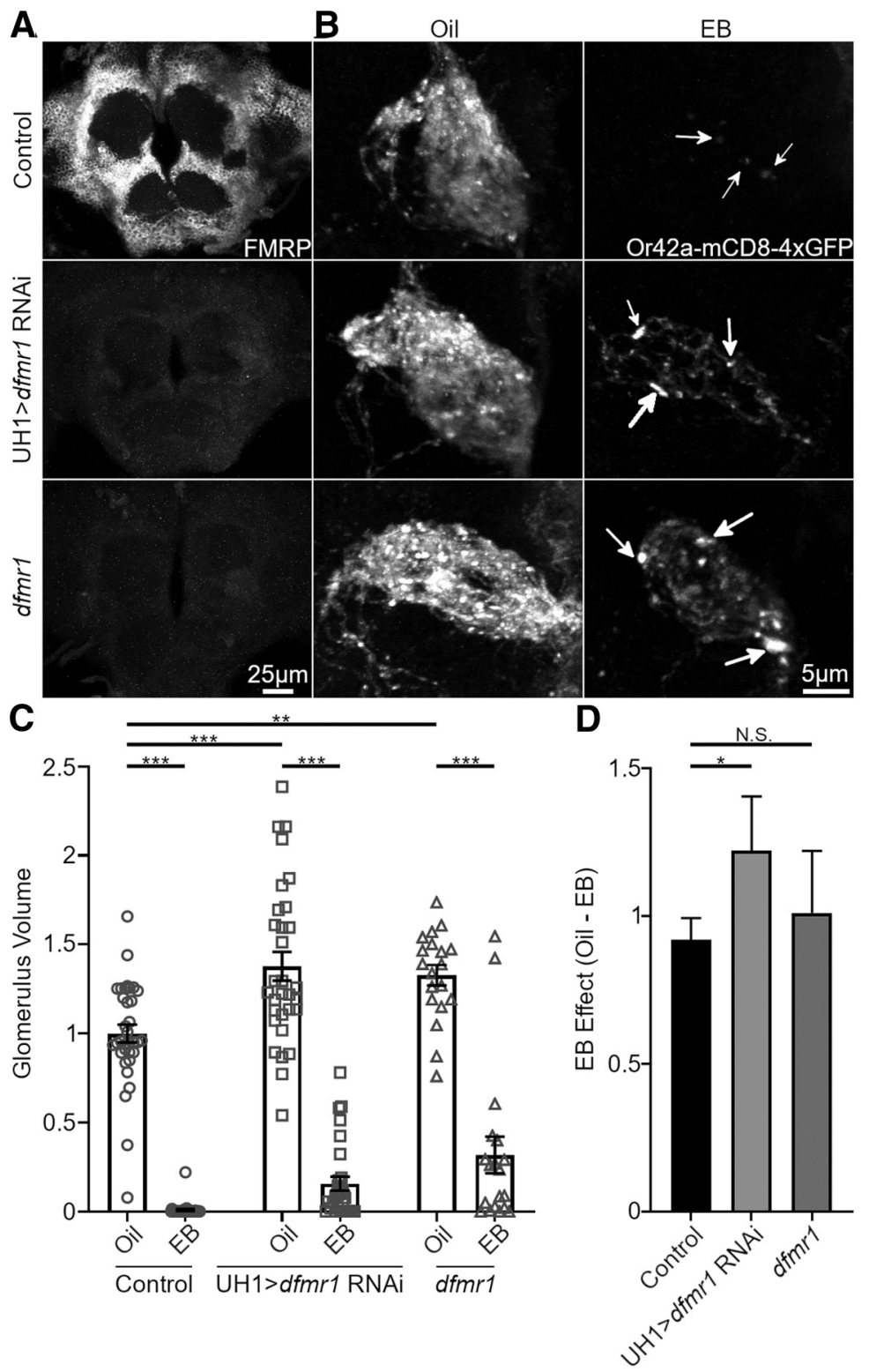

Figure 4. Neither dfmr1 mutants nor global dfmr1 RNAi impair OSN critical period remodeling $\boldsymbol{A}$, Representative confocal maximum intensity projections of the entire central brain labeled with anti-FMRP (white) in the w;Or42a-mCD8::4xGFP/ Or42a-mCD8::4xGFP;UH1-Gal4/+ transgenic control (top), with ubiquitous dfmr1 RNAi (w; Or42a-mCD8::4xGFP/Or42amCD8::4xGFP;UH1-Gal4/UAS-dfmr1 RNAi TriP GL00075; middle) and in a dfmr1 null mutant (dfmr ${ }^{B 55}$; bottom). B, Representative confocal maximum intensity projections of Or42a OSNs innervating the female AL VM7 glomerulus (Or42amCD8-4xGFP; white). The same genotypes above exposed to mineral oil vehicle (left) or 20\% EB odorant (right) from 0 to 2 dpe. The bright puncta following EB exposure are labeled by white arrows. C, Quantification of Or42a-0SN AL VM7 glomerulus innervation volumes comparing oil vehicle and $20 \% \mathrm{~EB}$ of all three genotypes. $\boldsymbol{D}$, The difference between oil and EB exposure for each genotype. The scatter plots show all data points and the mean \pm SEM for each assay. The bar graphs show mean \pm SER for each assay. The significance is indicated as not significant (N.S.; $p>0.05$ ), or significant at $* p<0.05$, $* * p<0.01, * * * p<0.001$.

ANOVA $(3 \times 2)$ analyses support the qualitative conclusion with significant effects of odor $\left(F_{(1,161)}=487.5, p=1.411 \mathrm{e}-50\right)$ and genotype $\left(F_{(2,161)}=18.08, p=8.252 \mathrm{e}-8\right)$, but no significant interaction $\left(F_{(2,161)}=2.57, p=0.0797\right.$; Fig. $\left.4 C\right)$. Pairwise $t$ tests with Sidak's correction indicate EB exposure significantly reduces innervation in all 3 genotypes [control oil $1.0 \pm 0.05(n=34)$ vs EB $0.008 \pm 0.006$ $(n=35) ; t_{(161)}=13.54, p=3.4 \mathrm{e}-27$; UH1-Gal4 $>d f m r 1$ RNAi oil $1.377 \pm 0.08(n=31)$ vs $\mathrm{EB} 0.156 \pm 0.04(n=29) ; t_{(161)}=15.52$, $p=1.27 \mathrm{e}-32 ; d f m r 1^{B 55}$ oil $1.328 \pm 0.06(n=19)$ vs $\mathrm{EB} 0.318 \pm 0.10$ $(n=19) ; t_{(161)}=10.23, p=4.62 \mathrm{e}-18$; Fig. $4 C$, bottom bars]. Vehicle 
null and RNAi animals both have larger basal innervation volumes (oil control vs oil UH1-Gal4 $>d f m r 1$ RNAi; $t_{(161)}=4.989, p=2.3 \mathrm{e}-5$; oil control vs $d f m r 1^{B 55}$ oil; $t_{(161)}=3.76, p=0.0036$; Fig. $4 C$, middle and top bars). Multiple linear regression to compare vehicle and $\mathrm{EB}$ exposure while controlling for this innervation difference shows UH1-Gal4 $>d f m r 1$ animals have a slightly larger change (UH1Gal4 $>$ dfmr1 RNAi x $20 \%$ EB $\beta=-0.23 \pm 0.11 ; t_{(161)}=2.127$, $p=0.035$; Fig. $4 D$, bottom bar) and $d f m r 1$ nulls have no significant difference $\left(d f m 1^{B 55} \times 20 \% \mathrm{~EB} \beta=-0.02 \pm 0.12 ; t_{(161)}=0.147\right.$, $p=0.8833$; Fig. $4 D$, top bar). Despite this small difference, both the ubiquitous FMRP knock-down and $d f m r 1^{B 55}$ mutant largely resemble the $d f m r 1^{50 M}$ mutant. These findings indicate that Or42a OSNtargeted FMRP removal selectively disrupts olfactory experience-dependent critical period remodeling. Together, these results further demonstrate that global FMRP loss in the AL circuit can be compensated for, despite the Or42a OSN-specific FMRP function.

\section{Or42a OSN-specific FMRP OE enhances critical period odorant remodeling}

The above results suggest that a FMRP balance between Or42a OSNs and other circuit neurons is required for proper critical period remodeling. If so, then elevating FMRP levels in OR42a OSNs should also alter experience-dependent remodeling. We have found bidirectional FMRP regulation within other neural circuits (Zhang et al., 2001; Sears et al., 2019). To test whether targeted FMRP OE impacts critical period remodeling, transgenic controls (Or42a>mCD8::GFP) were compared with UASFMRP 9557-3 (Or42a>FMRP OE; Zhang et al., 2001) after exposure to either the oil vehicle or 20\% EB during 0-2 dpe. As above, the controls show a striking reduction in Or42a OSN innervation (Fig. 5A, left panels, top vs bottom). In agreement with the FMRP balance hypothesis, Or42a OSN-targeted FMRP OE greatly enhances this olfactory experience-dependent remodeling (Fig. 5A, right panels, top vs bottom). Note that the directional disruption of the Or42a OSN innervation volume change is the opposite to Or42a OSN-targeted FMRP knock-down (Fig. 2 ), indicating a clear bidirectional consequence of FMRP imbalance within the circuit. Both the control and FMRP OE conditions show the OSN puncta characteristic of remodeling (Fig. $5 A$, arrows), but remnant Or42a OSN innervation is much sparser in the FMRP OE condition, similar to the consequence of increasing EB odorant concentration (Fig. 2). The quantitative assessment of Or42a OSN VM7 innervation volume further supports the role in FMRP balance in regulating olfactory experience-dependent synaptic remodeling during the early-use critical period.

ANOVA $(2 \times 2)$ analyses to compare these conditions show significant effects of both the genotype $\left(F_{(1,71)}=11.03\right.$, $p=0.0014)$ and odorant exposure $\left(F_{(1,71)}=186.7, p=1.471 \mathrm{e}-21\right)$, with a significant interaction between them $\left(F_{(1,71)}=13.96\right.$, $p=0.0004$; Fig. $5 B$ ). Unpaired $t$ tests with Sidak's correction pairwise comparisons show EB exposure in controls significantly reduces Or42a OSN innervation [control oil $1.0 \pm 0.037(n=17)$ vs $20 \%$ EB $0.48 \pm 0.054(n=17) ; t_{(71)}=6.714, p=2.35 \mathrm{e}-8$; Fig. $5 B$, bottom left], with a stronger effect following targeted FMRP OE [oil $1.022 \pm 0.07(n=21)$ vs EB $0.11 \pm 0.032(n=20) ; t_{(71)}=$ 12.92, $p=1.53 \mathrm{e}-19$; Fig. 5B, bottom right]. FMRP OE does not impact the basal innervation $\left(t_{(71)}=0.2955, p=0.9998\right)$, so we directly compared EB-exposed control and FMRP OE conditions. FMRP OE in the Or42a OSNs significantly enhances critical period remodeling from the EB exposure $\left(t_{(71)}=4.963\right.$, $p=2.75 \mathrm{e}-5$; Fig. $5 B$, top bar). This confirms the ANOVA analyses indicating a significant interaction between FMRP OE and
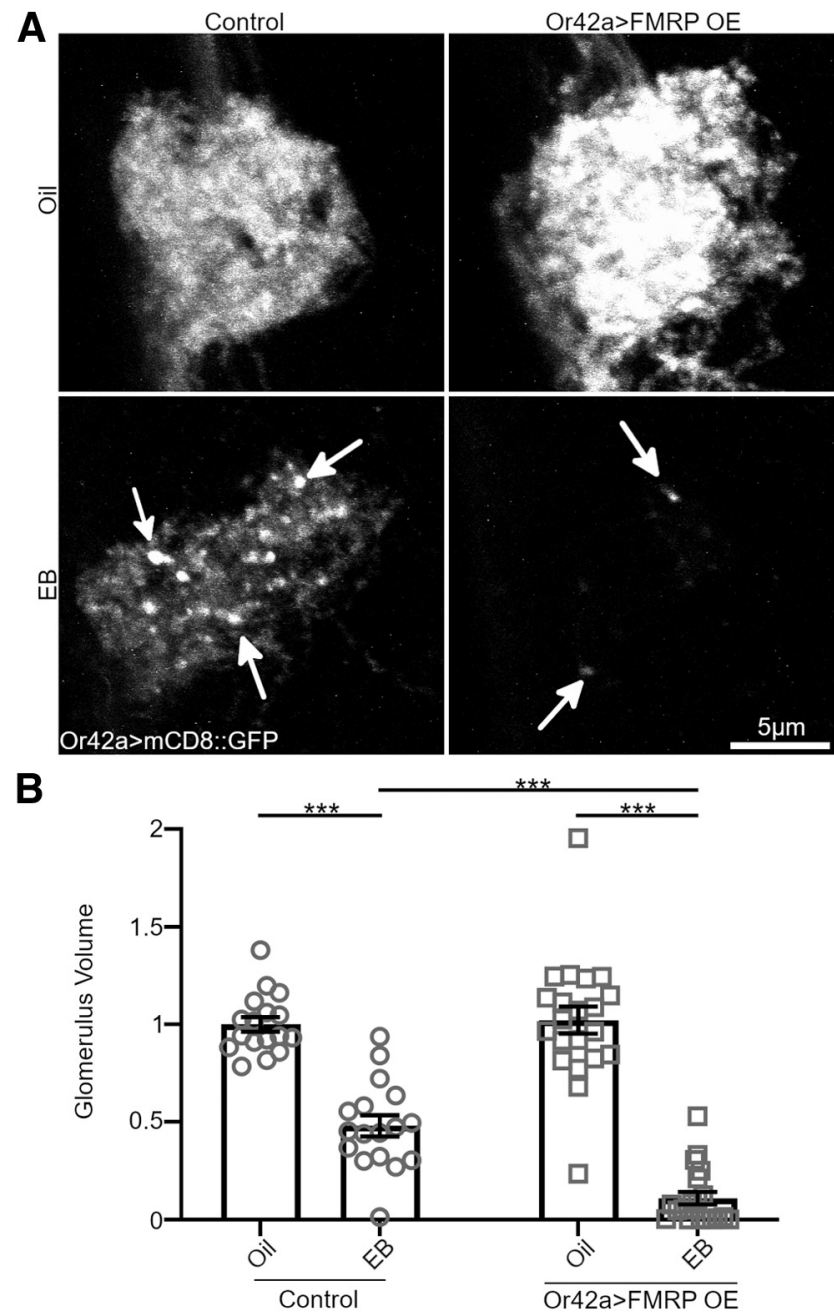

Figure 5. Or42a OSN-specific FMRP OE increases VM7 innervation remodeling $\boldsymbol{A}$, Representative confocal maximum intensity projections of Or42a OSNs innervating the $\mathrm{AL}$ VM7 glomerulus (Or42a-Gal4>UAS-mCD8::GFP; white). Females were exposed to mineral oil vehicle (top) or 20\% EB odorant (bottom) from 0 to 2 dpe. Two genotypes are shown: transgenic control (Or42a-Gal4>mCD8::GFP; left), and the same transgenic line overexpressing FMRP (Or42a-Gal4>FMRP 9557-3; right). The bright puncta following EB exposure are labeled by white arrows. $B$, Quantification of the Or42a-OSN VM7 innervation volume for both genotypes and treatment conditions. Scatter plots show all data points and the mean \pm SEM. The significance is indicated as $* * * p<0.001$.

odorant exposure. These results support the conclusion that FMRP balance within the AL circuit determines olfactory experience-dependent synaptic remodeling during the critical period. More specifically, the opposite effects of targeted FMRP decrease and increase only in Or42a OSNs indicates a bidirectional regulation of remodeling. When FMRP levels are lower in Or42a OSNs compared with other neurons, remodeling is diminished, and conversely increasing FMRP levels in Or42a OSNs enhances critical period remodeling.

\section{Pan-OSN FMRP knock-down does not impact olfactory experience critical period remodeling}

The above results indicate FMRP works cell autonomously and cell non-autonomously in opposition to regulate critical period olfactory experience OSN synaptic remodeling. Given the Or42a OSN-specific FMRP functions, the next goal was to identify the neurons providing the cell non-autonomous counterbalance. To begin this new pursuit, Or42a OSN remodeling was tested after 
altering FMRP levels in all OSNs. The OrcoGal4 line drives expression in all OSNs (Larsson et al., 2004) but comes on relatively late in pupation. Pebbled-Gal4 ( $\mathrm{Peb}-\mathrm{Gal} 4)$ is also expressed in all OSNs (Fig. 1E; Sweeney et al., 2007) and comes on earlier. Since both drivers include the Or42a OSNs, the prediction is that if only the Or42a OSNs are involved in the critical period remodeling, then altering FMRP levels should phenocopy the Or42a OSN-specific driver. Alternatively, if other OSNs contribute to Or42a OSN remodeling, then this should phenocopy the global UH1-Gal4, without affecting Or42a OSN remodeling. To test these two possibilities, Peb-Gal4 and OrcoGal4 were used to eliminate and overexpress FMRP throughout the OSN population while assaying specifically for Or42a OSN olfactory experience-dependent critical period remodeling. $\mathrm{Peb}-\mathrm{Gal} 4$ was first used to drive $d f m r 1$ RNAi, while labeling Or42a OSNs using Or42a-mCD8::4xGFP. Transgenic controls (lacking the RNAi) and experimental animals were again exposed to oil vehicle or $20 \% \mathrm{~EB}$ from 0 to 2 dpe. Sample images of FMRP expression in maxillary palp OSNs and Or42a OSN innervation in VM7 glomeruli, as well as quantified glomerular innervation values, are all shown in Figure 6.

All OSN cell bodies in the maxillary palp express FMRP, and can be co-labeled for the Or42a OSN population (Fig. 6A, top). PebGal4 driven FMRP RNAi (Peb-Gal4 $>d f m r 1$ RNAi) strongly suppresses FMRP in all OSNs, including Or42a OSNs (Fig. 6A, right). Following $\mathrm{EB}$ exposure, transgenic controls exhibit a near complete loss of Or42a OSN innervation (Fig. 6A, bottom). Because of strong loss of Or42a innervation (GFP signal) in the VM7 glomeruli, presynaptic active zone BRP labeling is also shown to outline the AL glomeruli (Fig. 6A, bottom, magenta). Three distinct glomeruli are thus demarcated, including the central VM7 glomerulus innervated by Or42a OSNs (Fig. $6 A$, dotted white lines). In the oil-exposed animals, typical innervation is observed. After 20\% EB critical period exposure, both transgenic controls and the $\mathrm{Peb}$ Gal4 $>d f m r 1$ RNAi animals show near complete loss of VM7 innervation (Fig. 6A, third row vs bottom). Importantly, Or42a OSN innervation loss corresponds with the loss of synaptic BRP within VM7 glomeruli, and therefore an inability to clearly define the VM7 border with BRP labeling (Fig. 6A, bottom, white dotted regions). This loss of BRP synapse after critical period EB exposure is consistent with our previous report that strong olfactory experience during the critical period diminishes BRP volume and intensity within Or42a OSN presynaptic active zones (Golovin et al., 2019). These results indicate that global OSN-targeted FMRP removal does

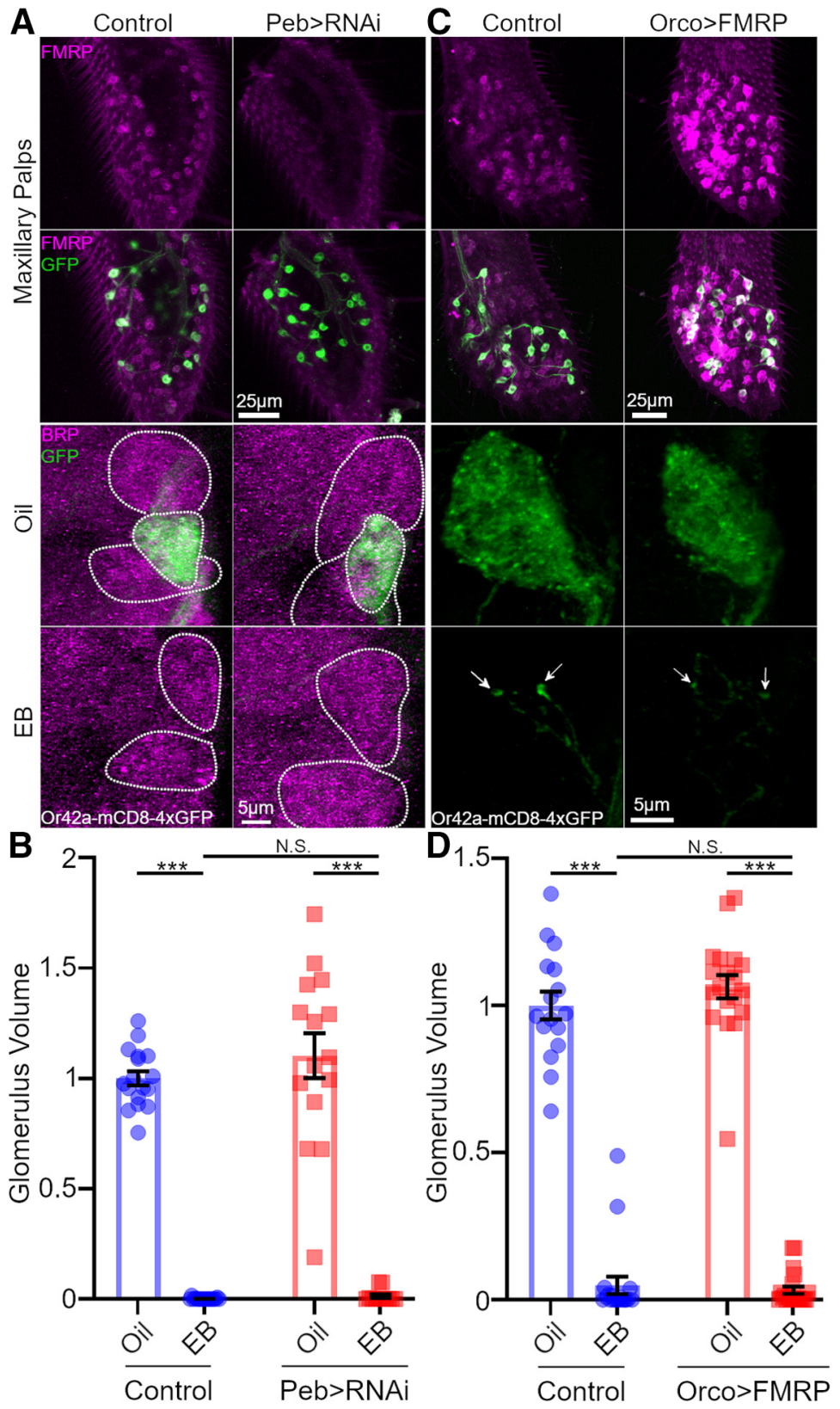

Figure 6. Pan-OSN FMRP knock-down/OE does not impact the VM7 remodeling $A$, Maxillary Palp (MP) anti-FMRP (magenta; top row), co-labeled with Or42a 0SNs (Or42a-mCD8-4xGFP, green; second row) in transgenic control (w;PebGal4/+;Or42a-mCD8::4xGFP/+;Or42a-mCD8::4xGFP/+; left) and Peb-Gal4>dfmr1 RNAi (w;:Peb-Gal4/+; Or42amCD8::4xGFP/+; Or42a-mCD8::4xGFP/UAS-dfmr1 RNAi; right). Bottom two rows, Or42a 0SN innervation of VM7 glomerulus after exposure to oil vehicle or 20\% EB from 0 to 2 dpe. BRP labeling (magenta) shows VM7 and surrounding glomeruli (dotted white outlines). $\boldsymbol{B}$, Quantification of Or42a OSN VM7 innervation volume with Peb-Gal4 dfmr1 RNAi. $\boldsymbol{C}$, The same MP labeling of transgenic control ( $w$; Or42a-mCD8::4xGFP/Or42a-mCD8::4xGFP; Orco-Gal4/+; left) and OrcoGal4 FMRP 0E (w; Or42a-mCD8::4xGFP/Or42a-mCD8::4xGFP; Orco-Gal4/UAS-FMRP 9557-3; right). Bottom two rows, The same Or42a OSN-VM7 innervation exposed to either oil vehicle or 20\% EB from 0 to 2 dpe. D, Quantification of the Or42a-OSN VM7 innervation volume for Orco-Gal4 FMRP OE. Scatter plot shows all data points and the mean \pm SEM. The significance is indicated as not significant (N.S.; $p>0.05$ ), or significant at $* * * p<0.001$.

not impact Or42a OSN innervation remodeling because of EB exposure during the critical period, a conclusion next confirmed by quantitative assessment.

ANOVA $(2 \times 2)$ analyses to compare genotypes versus odorant treatments show a significant effect of odorant $\left(F_{(1,57)}=\right.$ $378.3, p=7.734 \mathrm{e}-27)$, but not genotype $\left(F_{(1,57)}=1.148\right.$, $p=0.288)$, with no significant interaction between genotype and odorant $\left(F_{(1,57)}=0.7329, p=0.396\right.$; Fig. $\left.6 B\right)$. Unpaired $t$ tests 


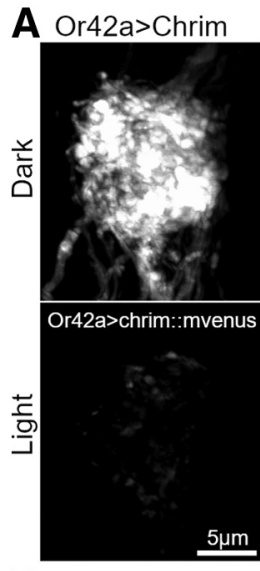

B
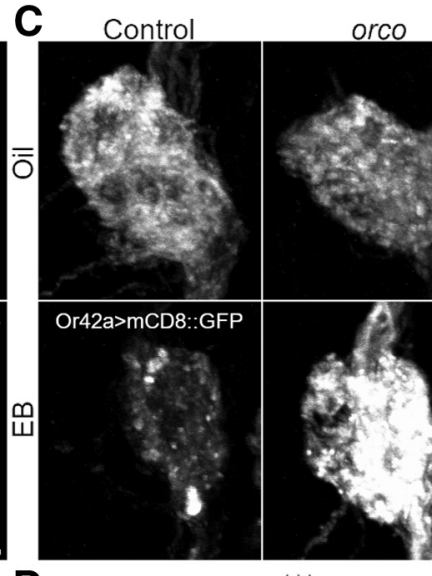

\section{D}

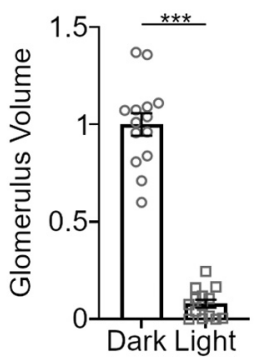

Or42a>Orco
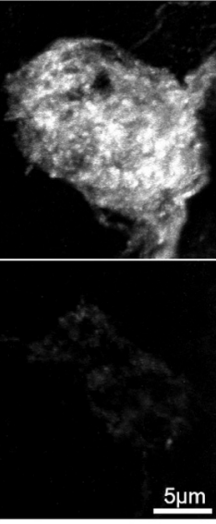

E

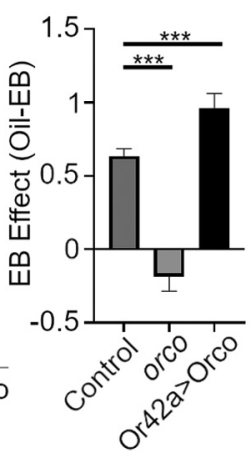

Figure 7. Or42a OSN-targeted neuronal activation drives VM7 critical period remodeling. $\boldsymbol{A}$, Representative confocal maximum intensity projections of Or42a-0SN VM7 innervation, with Or42a-Gal4 driven expression of fluorescently tagged channelrhodopsin Cschrimson::mVenus. Females were reared in complete darkness (dark, top) or with 515 nm cyan light (light, bottom) during the 0 - to 2-dpe critical period. B, Quantification of Or42a-0SN VM7 innervation volume without activation (dark) and with optogenetic stimulation (light). C, Representative images of Or42a OSN VM7 innervation (Or42a-Gal4>UAS-mCD8::GFP; white). Females were exposed to the oil vehicle (top) or 20\% EB (bottom) during the 0 - to 2-dpe critical period. Three genotypes are shown: transgenic control (w; UAS-mCD8:: $\mathrm{GFP} /+$; Or42a-Gal4/+; left), orco null mutant (orco ${ }^{7} / \mathrm{orco}^{2}$; middle), and the orco null with 0r42a 0SN-targeted Orco rescue (orco ${ }^{1}$ orco ${ }^{2}$, Or42a-Gal4>UAS-Orco; right). D, Quantification of VM7 innervation for all genotypes and conditions. $\boldsymbol{E}$, The difference between the oil vehicle and EB exposures. Scatter plots show all data points and mean \pm SEM. Bar graphs show mean \pm SER. The significance is indicated as significant at $* p<0.05$, $* * p<0.01, * * * p<0.001$.

with Sidak's correction evaluating pairwise comparisons indicate EB-exposed control animals have significantly reduced Or42a OSN innervation [control oil $1.0 \pm 0.032(n=17)$ vs $20 \% \mathrm{~EB}$ $0.002 \pm 0.001(n=17) ; t_{(57)}=14.02, p=4.64 \mathrm{e}-26$; Fig. $6 B$, bottom left]. FMRP OSN knock-down (Peb-Gal4 $>d f m r 1$ RNAi) also causes significantly reduced OSN innervation volumes following EB exposure $[P e b-G a l 4>d f m r 1$ RNAi oil $1.103 \pm 0.102$ $(n=15)$ vs EB $0.013 \pm 0.009(n=12) ; t_{(57)}=13.56, p=1.042 \mathrm{e}-$ 18; Fig. $6 B$, bottom right]. FMRP removal does not impact the basal extent of VM7 innervation $\left(t_{(57)}=1.408, p=0.6603\right)$, and we therefore can directly compare the two EB-exposed genotypes. Peb-Gal4>dfmr1 RNAi in all the OSNs does not significantly impact the Or42a OSN innervation following critical period EB exposure compared with transgenic controls $\left(t_{(57)}=\right.$ $0.1478, p>0.9999$; Fig. $6 B$, top bar). This confirms the above ANOVA analyses indicating no significant interaction between EB exposure and the Peb-Gal4 FMRP knock-down. These results bolster the hypothesis that balanced FMRP within the AL circuit acts to regulate Or42a OSN critical period remodeling, and predict that OE of FMRP in all OSNs should also have no effect, thus mirroring the results of panOSN knock-down.
We next examined FMRP in transgenic control and Orco-Gal4>FMRP maxillary palps to find strong FMRP OE in all OSNs, including the marked Or42a OSNs (Fig. 6C, top). Similar to global OSN FMRP knock-down, FMRP OE throughout OSNs does not impact Or42a OSN critical period remodeling following EB exposure (Fig. 6C, third and bottom rows, compare left and right). ANOVA $(2 \times 2)$ quantification confirms the similar EB effect between transgenic controls and Orco-Gal4>FMRP OE animals, with a significant main effect of odorant exposure $\left(F_{(1,71)}=907.8, p=3.473 \mathrm{e}-42\right)$, but not genotype $\left(F_{(1,71)}=0.518, p=0.474\right)$, and no significant interaction between EB exposure and genotype $\left(F_{(1,71)}=1.506, p=0.224\right.$; Fig. $\left.6 D\right)$. Pairwise comparisons using unpaired $t$ tests with Sidak's correction reveal that EB exposure significantly reduces VM7 glomerular innervation in both controls and with OrcoGal4>FMRP OE compared with the oilexposed animals [control oil $1.0 \pm 0.05(n=16)$ vs $20 \%$ EB $0.049 \pm 0.03(n=19) ; t_{(71)}=19.77$, $p=8.28 \mathrm{e}-30 ;$ Orco-Gal4 $>$ FMRP oil $1.06 \pm 0.04$ $(n=19)$ vs $20 \%$ EB $0.033 \pm 0.01(n=21) ; t_{(71)}=$ 22.98, $p=7.91 \mathrm{e}-34$; Fig. $6 D$, bottom bars]. In addition, these tests show that OrcoGal4>FMRP OE does not alter VM7 innervation after oil or EB exposure (control oil vs Orco-Gal4 $>$ FMRP oil; $t_{(71)}=1.331, p=0.712$; control 20\% EB vs Orco-Gal4>FMRP 20\% EB; $t_{(71)}=0.372, p=0.999$; Fig. $6 D$, top bar). These results, along with the results from global OSN FMRP RNAi, all point to a FMRP role in Or42a OSNs and other OSN classes controlling VM7 innervation remodeling driven by critical period olfactory experience.

Or42a OSN-specific optogenetic activation is sufficient to drive critical period remodeling The FMRP role across the OSN population suggests a function mediating Or42a OSN innervation remodeling in response to $\mathrm{EB}$ odorant exposure in the critical period. We previously showed that the functional Or42a receptor is required for Or42a OSN critical period remodeling (Golovin et al., 2019). However, EB activates other OSN classes (DoOR v2.0; Münch and Galizia, 2016), and higher EB levels may activate more OSNs (Semmelhack and Wang, 2009). Therefore, it remains possible that critical period remodeling requires both Or42a receptors and EB-sensitive receptors in other OSN classes. To test this hypothesis, parallel approaches were employed. First, CsChrimson::mVenus channelrhodopsin (Klapoetke et al., 2014) was targeted to Or42a OSNs (Or42aGal4>CsChrimson:: $m$ Venus) for the specific activation of Or42a OSNs with timed cyan $(515 \mathrm{~nm})$ light stimulation (Fig. 7A,B). Second, removal of the essential olfactory Orco co-receptor required to mediate OSN responses (Larsson et al., 2004) was used to compare orco null mutants and orco nulls with Orco reexpressed only within Or42a OSNs, compared with transgenic driver controls (Fig. 7C). These tests allow the assessment of (1) the requirement of OSN activity in general, and (2) the sufficiency of Or42a OSN activity specifically, to mediate critical period remodeling. As above, Or42a OSN innervation was imaged 
within the VM7 glomerulus following 0- to 2-dpe critical period exposure to either the oil vehicle or $20 \%$ EB. Representative images and innervation quantifications for both channelrhodopsin and orco mutant experiments are shown in Figure 7.

To mimic the effects of EB odorant exposure during the critical period, Or42a-Gal4>CsChrimson::mVenus animals were staged and exposed to $515 \mathrm{~nm}$ light as closely as possible to the manner of EB odorant exposure. However, there were two differences: (1) the animals were kept in constant darkness before exposure to cyan light stimulus; and (2) the animals were raised in Petri dishes to allow for stronger optogenetic light stimulation (see Materials and Methods). Critical period light exposure of the targeted Or42a OSN CsChrimson-expressing animals drives a striking reduction of the Or42a innervation of the VM7 glomerulus, which qualitatively resembles the remodeling driven by the critical period EB olfactory experience (Fig. $7 A$, top vs bottom). Changes include a reduction in intensity, sparse and reduced glomerulus coverage, and appearance of the characteristic OSN puncta. This qualitative assessment is supported by quantitative measurements, which show that Or42aGal4>CsChrimson::mVenus animals exposed to cyan light during the critical period have significantly reduced innervation compared with dark-reared control animals (dark $1.0 \pm 0.058$ vs light $0.08 \pm 0.02 ; t_{(26)}=15.04 ; p=2.421 \mathrm{e}-14$, unpaired $t$ test; Fig. $7 B$ ). The ability of channelrhodopsindriven activity to reduce Or42a OSN innervation similar to olfactory EB exposure indicates that Or42a activity is sufficient for critical period synaptic remodeling of VM7 glomerulus innervation. However, because light and odorant driven activity levels in the Or42a neurons could be different, it is possible that the magnitude of innervation remodeling is different when only Or42a OSNs are activated.

\section{Or42a OSN-specific odorant activation is essential for critical period innervation remodeling}

To test whether Or42a OSN-specific EB receptor activation produces the same effect as global OSN EB activation, orco null mutants were compared with orco nulls with targeted Orco rescue only in Or42a OSNs (Or42a-Gal4>UAS-Orco; Fig. 7C). As in all the above experiments, the transgenic controls show the normal striking reduction in VM7 innervation caused by $20 \%$ EB odorant exposure during the 0 - to 2-dpe critical period, compared with the robust innervation characterizing the oil vehicle alone (Fig. 7C, left, top vs bottom). Consistent with the role of olfactory reception mediating critical period remodeling (Golovin et al., 2019), orco null mutants lack any reduction in VM7 innervation following critical period $\mathrm{EB}$ exposure, compared with the matched oil-exposed animals (Fig. 7C, middle, top vs bottom). In agreement with the above optogenetic studies, when Orco is re-expressed only in Or42a OSNs (Or42a-Gal4>Orco) there is again a strong reduction in VM7 innervation following EB olfactory experience during the critical period, compared with the oilexposed animals (Fig. 7C, right, top vs bottom). Interestingly, the reduction of VM7 innervation with targeted Or $42 a-G a l 4>$ Orco rescue appears even more extreme than the matched control animals (Fig. $7 C$, left bottom vs right bottom). In the targeted Or $42 a-G a l 4>$ Orco rescue animals, critical period EB exposure generates sparser and less intensely labeled VM7 glomeruli innervation. Taken together, these results suggest a highly specific Or42a OSN activity requirement in critical period innervation remodeling, a conclusion supported and expanded by quantitative analyses.
ANOVA $(3 \times 2)$ quantification comparing the controls and orco null mutants exposed to either EB or oil vehicle alone during the 0 - to 2-dpe critical period shows significant effects of genotype $\left(F_{(2,105)}=76.63 p=3.016 \mathrm{e}-21\right)$ and odorant exposure $\left(F_{(1,105)}=266.3, p=1.455 \mathrm{e}-30\right)$, with a significant interaction between the two $\left(F_{(2,105)}=142.3, p=1.273 \mathrm{e}-30\right.$; Fig. $\left.7 D\right)$. Unpaired $t$ tests with Sidak's correction evaluating pairwise comparisons show EB-exposed transgenic controls (Or42aGal4>mCD8::GFP) have significantly reduced innervation volumes [control oil $1.0 \pm 0.025(n=20)$ vs EB $0.365 \pm 0.045$ $(n=17) ; t_{(105)}=12.69, p=9.46 \mathrm{e}-22$; Fig. $7 D$, bottom left bar]. Unexpectedly, the oil-exposed orco null mutants (orco $1 /$ orco $^{2}$, Larsson et al., 2004) exhibit significantly lower innervation volumes compared with matched controls [control oil vs orco oil $0.842 \pm 0.032(n=19) ; t_{(105)}=3.245, p=0.0234$; Fig. $7 D$, second from bottom bar]. Although the orco null AL has been reported to have grossly normal overall morphology (Larsson et al., 2004), this basal difference could mark the beginnings of the later glomerular degeneration that later occurs in orco null mutants (Chiang et al., 2009). Also surprisingly, orco null mutants exposed to $20 \%$ EB during the critical period have significantly larger glomerulus innervation volumes than the oilexposed orco mutants [orco oil vs orco EB $1.028 \pm 0.041$ $(n=20) ; t_{(105)}=3.814, p=0.0035$; Fig. $7 D$, bottom middle bar]. These results show that complete loss of olfaction in all the ORexpressing OSNs has striking impacts on the olfactory circuitry and may shift the AL circuit connectivity in unexpected ways.

Since the oil-exposed control animals and EB-exposed orco mutants show no significant difference in VM7 glomerulus innervation $\left(t_{(105)}=0.577, p>0.9999\right)$, one possible explanation is that $\mathrm{EB}$ exposure prevents the glomerular degeneration starting to appear in orco mutants via the lateral excitation onto Or42a OSNs triggered by other OSN classes (Huang et al., 2010). Despite the above unanticipated results, the orco null mutants clearly show the expected lack of VM7 innervation remodeling after critical period EB exposure (Fig. $7 C, D$ ). There is a significantly increased innervation volume in orco nulls exposed to EB, compared with EB-exposed controls $\left(t_{(105)}=13.25, p=5.71 \mathrm{e}-23\right.$; Fig. $7 D$, third from bottom bar). Targeted restoration of Orco only in Or42a OSNs in otherwise orco null mutants prevents the innervation loss occurring in the orco nulls alone, leading to innervation volumes that are not significantly different compared with the oil-exposed controls [control oil = vs Or42aGal4>Orco oil $0.983 \pm 0.043(n=18) ; t_{(105)}=0.3539, p>0.9999$; Fig. $7 D$ ]. The Or42a-Gal $>$ Orco rescue condition also restores the VM7 innervation remodeling caused by critical period EB exposure, compared with the oil vehicle condition alone [EB $0.02 \pm 0.015(n=17) ; t_{(105)}=18.77, p=3.74 \mathrm{e}-34$; Fig. $7 D$, bottom right bar]. Moreover, the VM7 innervation reduction is significantly greater than within EB-exposed control animals $\left(t_{(105)}=\right.$ 6.636, $p=2.17 \mathrm{e}-8$; Fig. $7 D$, top bar). These results demonstrate that Orco-dependent Or42a OSN activation is the main driver of critical period AL innervation remodeling, but that other OSNs can fine-tune the response.

To directly compare interactions between critical period EB exposure and each genotype separately, a linear regression model was used to generate interaction terms interrogated to determine whether genotypes significantly alter innervation following EB exposure (Fig. 7E). There is a significant interaction between the critical period EB exposure and the orco null mutant genotype, with a significant regression coefficient $(20 \% \mathrm{~EB} \times$ orco $\beta=$ $0.82 \pm 0.07 ; t_{(105)}=11.76, p=7.082 \mathrm{e}-21$; Fig. $7 E$, left bar). This result indicates that introduction of the orco null prevents the 
olfactory experience-dependent innervation loss after critical period $\mathrm{EB}$ exposure, compared with the matched controls. The interaction of the odorant exposure with the Or42a-Gal4>Orco rescue in an otherwise orco null mutant is in the opposite direction, with another very significant regression coefficient $(20 \%$ EB $\mathrm{x}$ Or42a $>$ Orco $\beta=-0.328 \pm 0.071 ; t_{(105)}=4.574$, $p=1.315 \mathrm{e}-5$; Fig. $7 E$, right bar). This result indicates that the critical period EB odorant exposure leads to a greater reduction of VM7 innervation volume in the orco null mutants with Orco reexpressed only in the Or42a OSNs, compared with the transgenic control animals. Taken together, these results provide very strong evidence that Or42a OSN-specific activity is sufficient to drive critical period remodeling of the VM7 glomerulus innervation. However, although only Or42a OSN activity is required for the innervation remodeling, other OSNs appear to modulate the level of EB experience remodeling through lateral inhibition, as the innervation reduction seen when Orco is only targeted to Or42a OSNs is greater than in the matched transgenic control animals.

\section{Or42a OSN-targeted activation is not affected by Or42a OSN-targeted FMRP knock-down}

The Or42a OSN presynaptic terminals innervating the VM7 glomerulus receive lateral inhibition from AL GABAergic LNs (Fig. $1 E$, bottom left; Olsen and Wilson, 2008). This inhibition scales with OSN olfactory activation and is effectively blocked by removing or shielding from external odorant stimuli (Olsen and Wilson, 2008). Based on the role of balanced OSN FMRP levels (Fig. 6), and the impact of silencing many OSNs (Fig. 7) in mediating the Or42a OSN critical period remodeling, we next hypothesized that FMRP might regulate the local lateral modulation downstream of broad EB-driven OSN activation. To test this hypothesis, we took advantage of Or42a OSN-targeted CsChrimson::mVenus channelrhodopsin (Klapoetke et al., 2014) to specifically activate just the target OSNs (as in Fig. 7), while also targeting FMRP removal (Or42a-Gal4>UAS-CsChrimson:: mVenus, UAS-dfmr1 RNAi; Fig. 8). These animals were compared with animals raised in total darkness, and to transgenic control animals (lacking the RNAi) raised in either darkness or in the same light conditions. As further controls, we examined the effect of oil vehicle alone or $20 \%$ EB on the same genotypes raised in complete darkness. All light and odorant treatments were done in the 0 - to 2 -dpe critical period. Representative images of Or42a OSN terminals in the VM7 glomerulus, and the innervation quantifications for all genotypes and treatments, are shown in Figure 8.

Transgenic controls show the expected strong reduction in Or42a OSN innervation of the VM7 glomerulus following critical period light stimulation, compared with animals raised in total darkness (Fig. 8A). Or42a OSN-specific optogenetic activation in $\mathrm{Or} 42 a-\mathrm{Gal} 4>\mathrm{UAS}-d f m r 1$ RNAi animals causes a similar decrease in VM7 innervation compared with the dark-reared animals (Fig. $8 A$ vs $B$ ), indicating the EB-driven OSN activity is an important driver of the FMRP effect. ANOVA $(2 \times 2)$ analyses of glomeruli innervation for each condition show a significant light stimulation effect $\left(F_{(1,73)}=114.3, p=1.368 \mathrm{e}-16\right)$, but no FMRP genotype effect $\left(F_{(1,73)}=0.05, p=0.8158\right)$, with no significant interaction between stimulation and genotype $\left(F_{(1,73)}=\right.$ $1.71, p=0.195)$. Pairwise comparisons using $t$ tests with Sidak's correction show that critical period EB exposure significantly reduces innervation in both transgenic controls and Or42aGal4>UAS-dfmr1 RNAi animals [control dark $1.0 \pm 0.04$ $(n=21)$ vs light $0.41 \pm 0.06(n=21) ; t_{(73)}=8.9, p=1.71 \mathrm{e}-12$;

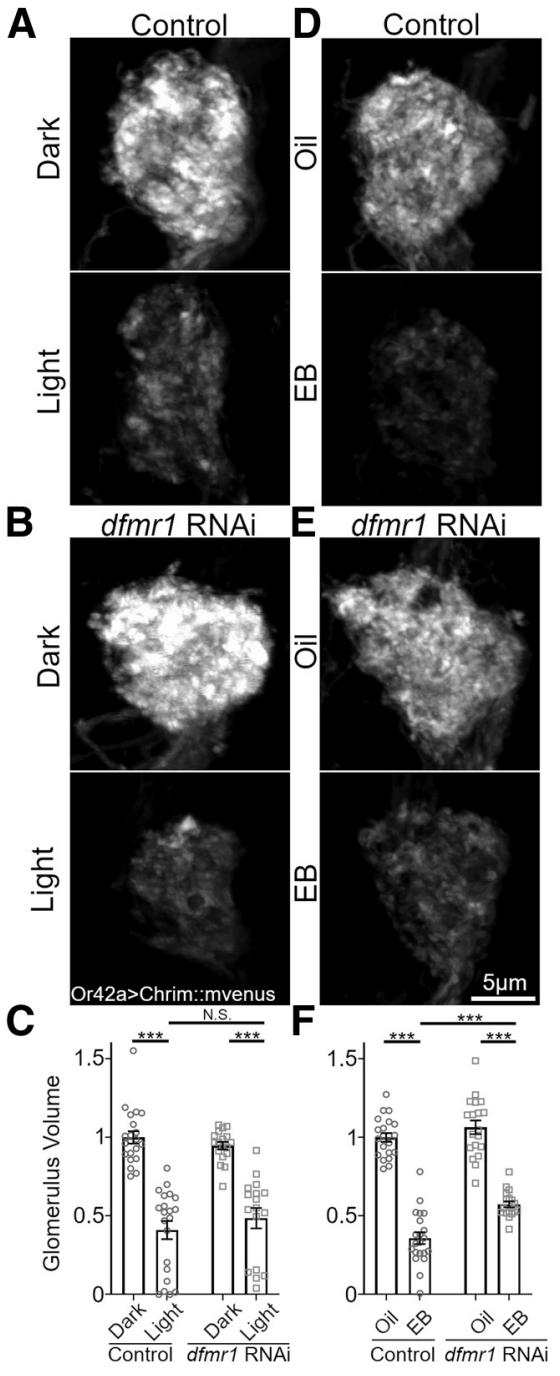

Figure 8. Or42a-targeted optogenetic activation is not affected by Or42a-targeted FMRP RNAi All images show confocal maximum intensity projections of Or42a-0SN VM7 innervation, with Or42a-Gal4 driven expression of fluorescently tagged channelrhodopsin Cschrimson::mVenus. $\boldsymbol{A}, \boldsymbol{B}$, Females were reared in total darkness (dark, top) or with $515 \mathrm{~nm}$ cyan light (light, bottom). $\boldsymbol{A}$, Or42a $>$ Cschrimson::mVenus transgenic controls and (B) Or42atargeted dfmr1 RNAi (TriP GL00075). C, Quantification of VM7 innervation in control and Or42a $>$ dfmr1 RNAi animals following dark and light treatment. $\boldsymbol{D}, \boldsymbol{E}$, Females were reared with oil vehicle (top) or $20 \%$ EB odorant (bottom) during the 0 - to 2-dpe critical period. $\boldsymbol{D}$, Or42a $>$ Cschrimson::mVenus transgenic controls and $(\boldsymbol{E})$ Or42a-targeted dfmr1 RNAi (TriP GL00075). $\boldsymbol{F}$, Quantification of VM7 innervation in control and 0r42a $>$ dfmr1 RNAi animals following oil and 20\% EB exposure. Scatter plots show all data points and the mean \pm SEM. The significance is indicated as not significant (N.S.; $p>0.05$ ) and significant at $* * * p<0.001$.

RNAi dark $0.947 \pm 0.02(n=18)$ vs light $0.486 \pm 0.07(n=17)$; $t_{(73)}=6.35, p=9.7 \mathrm{e}-8$; Fig. $8 C$, bottom bars]. Further comparisons show that transgenic controls raised in darkness or lightstimulated during the 0 - to 2 -dpe critical period have VM7 glomerulus innervation volumes not statistically different from Or42a-Gal4>UAS-dfmr1 RNAi animals under the same conditions (control dark vs RNAi dark; $t_{(73)}=0.766, p=0.9712$; control light vs RNAi light; $t_{(73)}=1.08, p=0.8641$; Fig. $8 C$, top bar). Together, these results indicate that broad OSN activation is required for the effect of Or42a OSN-targeted FMRP removal.

Consistent with previous results, transgenic controls exposed to $20 \%$ EB show reduced Or42a OSN VM7 innervation compared with oil-exposed animals (Fig. $8 D$, top vs bottom). The 
effect of Or42a OSN-targeted FMRP RNAi also agrees with previous experiments, showing impaired innervation remodeling compared with the transgenic controls (Fig. $8 E$ ). ANOVA $(2 \times 2)$ quantification of the effects of oil versus $20 \%$ EB exposure on the two genotypes show a significant effect of both odorant $\left(F_{(1,75)}=\right.$ 284.1, $p=3.211 \mathrm{e}-27)$ and genotype $\left(F_{(1,75)}=\right.$ 17.3, $p=8.4 \mathrm{e}-5)$, with a significant interaction between exposure and genotype $\left(F_{(1,75)}=5.02\right.$, $p=0.028$ ). Pairwise comparisons using $t$ tests with Sidak's correction show that EB exposure significantly reduces VM7 glomerulus innervation volume for both transgenic controls and Or42a-Gal4>UAS-dfmr1 RNAi animals [control oil $1.0 \pm 0.03 \quad(n=21) \quad$ vs $20 \% \quad \mathrm{~EB}$ $0.359 \pm 0.04(n=21) ; t_{(75)}=13.95, p=9.38 \mathrm{e}-22$; RNAi oil $1.064 \pm 0.04 \quad(n=19)$ vs $20 \%$ EB $0.574 \pm 0.02(n=18) ; t_{(75)}=10.02, p=1.05 \mathrm{e}-14$; Fig. $8 F$, bottom bars]. Or $42 a-G a l 4>$ UAS-dfmr 1 RNAi animals exposed to the oil vehicle have statistically similar glomerulus innervation volumes to the transgenic controls (control oil vs RNAi oil; $\left.t_{(75)}=1.37, p=0.687\right)$. In line with our previous experiments, Or $42 a-G a l 4>$ UAS$d f m r 1$ RNAi animals had significantly greater VM7 innervation compared with transgenic controls after critical period EB exposure (control EB vs RNAi EB; $t_{(75)}=4.49, p=0.0002$; Fig. $8 F$, top bar). Overall, these results suggest lateral connections from broadly branching AL LNs are the likely mediators of critical period remodeling.

\section{Or42a OSN synaptic output is not required for Or42a-targeted FMRP RNAi remodeling effects}

Results up to this point indicate that Or42a

OSN-specific activity is needed for critical period remodeling and that refinement is impaired if FMRP levels are not balanced between OSNs. We previously reported that Or42a OSN synaptic output does not drive critical period remodeling, but rather serves to limit the effect of EB odorant experience (Golovin et al., 2019). Because of the known FMRP roles controlling trans-synaptic signaling that coordinates with neurotransmitter release (Friedman et al., 2013), we hypothesized that FMRP roles in critical period remodeling require synaptic output. To block synaptic output in Or42a OSNs, we used a targeted tetanus toxin light chain (Or42a-Gal4>UAS-TeTxLc), while unbalancing FMRP levels using targeted FMRP RNAi (Or42a-Gal4>UAS-dfmr1 RNAi), with Or42a-Gal4>UAS-mCD8::GFP labeling. The combined Or42a-Gal4>UAS-TeTxLc, UAS-dfmr1 RNAi animals were compared with the controls (Or42a-Gal4>UAS-mCD8::GFP), as well as TeTxLc (Or42a-Gal4>UAS-TeTxLc) and FMRP RNAi (Or42a-Gal4>UAS-dfmr1 RNAi) alone. Animals of each genotype were exposed to either the oil vehicle or $10 \%$ EB during the 0 - to 2-dpe critical period. The lower EB concentration was used for this experiment because our previous work with Or42aGal4>UAS-TeTxLc animals showed that Or42a OSN innervation of the VM7 glomerulus was completely eliminated at higher EB concentrations (Golovin et al., 2019). Representative images of Or42a OSN terminals in the VM7 glomerulus, innervation quantifications, and EB effect quantifications for all genotypes and treatments are shown in Figure 9.

Unlike the higher EB exposures, 10\% EB from 0 to 2 dpe has little effect on Or42a OSN innervation in transgenic control animals (Fig. 9A, top), although characteristic OSN punctae still occur after odorant exposure. The 10\% EB-exposed controls show some regions of thinner VM7 innervation, with other areas containing the OSN puncta often occurring in EB-exposed animals (Fig. 9A, top, right arrows). Comparing Or42a-Gal4>UASdfmr1 RNAi animals exposed to oil vehicle or EB odorant also reveals similar OSN puncta (Fig. 9A, second row, right arrows). However, unlike the control animals, Or42a-target FMRP RNAi animals have more widespread OSN puncta with little thinning of the innervation, which leads to a small expansion of the overall glomerulus innervation (Fig. 9A, second row). Consistent with our previous experimental report (Golovin et al., 2019), Or42aGal4 $>$ UAS-TeTxLc causes both more expansive basal innervation and a stronger EB odorant-induced reduction than matched controls (Fig. 9A, third row). These TeTxLc-expressing animals show a response to critical period $10 \% \mathrm{~EB}$ exposure that appears similar to the control response to $20 \%$ EB. In addition, examining animals expressing both Or42a-targeted $d f m r 1$ RNAi and TeTxLc blockade shows basal glomerulus innervation even further increased compared with controls (Fig. 9A, bottom row). Moreover, EB-exposed animals with both $d f m r 1$ RNAi and 
TeTxLc synapse blockade show impaired remodeling compared with TeTxLc alone (Fig. 9A, bottom right). These results indicate that blocking Or42a OSN synaptic output does not impair the effect of Or42a-targeted FMRP RNAi.

ANOVA $(2 \times 2 \times 2)$ quantitative analyses of VM7 glomerulus innervation for each condition strongly support the above conclusions (Fig. 9B,C). Comparisons show a significant effect of the EB odorant exposure $\left(F_{(1,316)}=335.9, p=1.272 \mathrm{e}-51\right)$, TeTxLc synaptic transmission blockade $\left(F_{(1,316)}=34.04, p=1.336 \mathrm{e}-8\right)$ and targeted $d f m r 1$ RNAi $\left(F_{(1,316)}=102.4, p=4.924 \mathrm{e}-21\right)$, with significant two-way interactions between odorant and TeTxLc $\left(F_{(1,316)}=428.4, p=9.46 \mathrm{e}-61\right)$, odor and $d f m r 1 \operatorname{RNAi}\left(F_{(1,316)}=\right.$ 15.9, $p=8.3 \mathrm{e}-5)$, and TeTxlc and $d f m r 1$ RNAi $\left(F_{(1,316)}=7.96\right.$, $p=0.0051)$, but no significant three-way interaction $\left(F_{(1,316)}=\right.$ $0.0014, p=0.9697)$. Pairwise comparisons using $t$ tests with Sidak's correction show that critical period EB exposure does not significantly change VM7 glomerulus innervation for the transgenic controls compared with the oil-exposed animals [control oil $1.0 \pm 0.03(n=42)$ vs $10 \%$ EB $0.959 \pm 0.03(n=41) ; t_{(316)}=$ $0.8375, p>0.9999$; Fig. $9 B$, bottom left bar]. Interestingly, Or42a-Gal4 > UAS-dfmr1 RNAi animals have significantly larger VM7 glomerulus innervation volumes after EB exposure compared with the oil vehicle [RNAi oil $1.082 \pm 0.04(n=39)$ vs $10 \%$ EB $1.242 \pm 0.04(n=41) ; t_{(316)}=3.179, p=0.0445$; Fig. $9 B$, second bottom bar]. Critical period $10 \%$ EB exposure in Or $42 a$ OSN-targeted TeTxLc animals, with or without $d f m r 1$ RNAi, leads to a significant reduction in VM7 innervation compared with the oil-exposed animals [TeTxLC oil $1.3 \pm 0.03(n=41)$ vs $10 \%$ EB $0.226 \pm 0.03 \quad(n=38) ; t_{(316)}=21.22, p=2.69 \mathrm{e}-61$; $\mathrm{RNAi}+\mathrm{TeTxLc}$ oil $1.525 \pm 0.04(n=42)$ vs $10 \% \mathrm{~EB} 0.648 \pm 0.05$ $(n=40) ; t_{(316)}=17.65, p=1.5 \mathrm{e}-47$; Fig. $9 B$, bottom third and right bars].

Further comparisons show that oil-exposed transgenic controls have similar Or42a OSN VM7 innervation compared with Or42a-Gal4>UAS-dfmr1 RNAi, but significantly smaller than with Or42a-Gal4>UAS-TeTxLc (control oil vs RNAi oil; $t_{(316)}=$ 1.636, $p=0.9519$; control oil vs TeTxLc oil; $t_{(316)}=6.079$, $p=9.75 \mathrm{e}-8$; Fig. $9 B$, second and third from bottom left bars). Combining both $d f m r 1$ RNAi and TeTxLc significantly increases the glomerulus innervation of oil-exposed animals, possibly indicating an odor experience-independent interaction (TeTxLc oil vs dfmr1 RNAi + TeTxLc oil; $t_{(316)}=4.553, p=0.0002$; Fig. $9 B$, second from bottom bar right). As expected, following $10 \% \mathrm{~EB}$ exposure, Or42a OSN-targeted $d f m r 1$ RNAi impairs and Or $42 a$ OSN-targeted TeTxLc enhances VM7 glomerulus innervation, compared with matched controls (control $10 \%$ EB vs RNAi $10 \%$ $\mathrm{EB} ; t_{(316)}=5.7, p=7.69 \mathrm{e}-7$; control $10 \% \mathrm{~EB}$ vs TeTxLc $10 \% \mathrm{~EB}$; $t_{(316)}=14.47, p=2.58 \mathrm{e}-35$; Fig. $9 B$, fourth from bottom and top left bars). Glomerulus innervation was also compared in Or42aGal4>UAS-TeTxLc animals after 10\% EB exposure, with or without $d f m r 1$ RNAi. Similar to Or42a OSNs with intact synaptic output, transmission-blocked animals with $d f m r 1$ RNAi show significantly increased VM7 innervation, implying the remodeling impairment from imbalanced FMRP levels does not require synaptic transmission from Or42a OSNs (TeTxLC 10\% EB vs RNAi + TeTxLc 10\% EB; $t_{(316)}=8.289, p=9.265 \mathrm{e}-14$; Fig. $9 B$, right, fourth bar from bottom). The ANOVA quantification indicates that FMRP effects Or42a OSN critical period remodeling independent of OSN synaptic output.

To compare interactions between the critical period EB exposure and each of the genotypes, a linear regression model was generated to test for significant interactions (Fig. 9C). There is significant interaction between critical period EB experience and targeted $d f m r 1$ RNAi, with a significant regression coefficient $\left(10 \%\right.$ EB $\mathrm{x}$ dfmr1 RNAi $\beta=0.22 \pm 0.07 ; t_{(316)}=3.108$, $p=0.0021$; Fig. $9 C$, bottom left bar), indicating that targeted $d f m r 1$ RNAi significantly mitigates EB-induced innervation remodeling. The interaction of critical period EB exposure and TeTxLc is in the opposite direction, with another very significant regression coefficient $(10 \% \mathrm{~EB} \times \mathrm{TeTxLc} \beta=-1.009 \pm 0.07$; $t_{(316)}=13.96, p=7.849 \mathrm{e}-35$; Fig. $9 C$, top bar), indicating that EB exposure causes a greater reduction of VM7 innervation in Or42a-targeted TeTxLc animals. The interaction between EB exposure and targeted $d f m r 1$ RNAi is not altered by the TeTxLc blockade (10\% EB x dfmr1 RNAi x TeTxLc $\beta=-0.027 \pm 0.1$; $\left.t_{(316)}=0.26, p=0.795\right)$. To test more specifically for an effect of Or42a-targeted $d f m r 1$ RNAi on EB exposure in the TeTxLc animals, we built a second linear regression model using only the TeTxLc blockade data. The regression coefficient for the interaction between critical period EB exposure and Or42a OSN-targeted $d f m r 1$ RNAi in the TeTxLc model is still significant $(10 \%$ EB x dfmr1 RNAi $\beta=0.1973 \pm 0.07 ; t_{(157)}=2.77, p=0.0063$; Fig. $9 C$, bottom right bar). Taken together, these findings indicate that the cell autonomous FMRP role on odorant experiencedependent innervation remodeling does not rely on the Or42a OSN synaptic output.

It is possible that OSN-targeted TeTxLc synaptic transmission blockade might be having effects by modifying FMRP levels in these neurons. In the above FMRP OE studies, elevating FMRP levels in the Or42a OSNs enhances the effect of EB experiencedependent critical period remodeling (Fig. 4). Therefore, rather than TeTxLc blockade acting independently from FMRP, OSNtargeted TeTxLc could possibly increase FMRP expression and thus mimic the effects of FMRP OE. In order to test this possibility, we used FMRP antibody labeling to compare controls to animals expressing TeTxLc in Or42a OSNs. Silencing synaptic output of Or42a OSNs does not detectably alter FMRP levels in these neurons (Fig. 10). Comparisons of Or42a OSN somata in maxillary palps reveal no differences in the intensity or extent of FMRP labeling (Fig. 10A). Quantification shows that Or42a OSN FMRP levels do not significantly differ between the controls and Or42a-Gal4>TeTxLc animals [control $2723 \pm 161.4$ FMRP intensity (A.U.; $n=20$ palps) vs Or42a-Gal4 $>$ TeTxLc $2445 \pm 417.8$ arbitrary units (A.U.) $(n=12)$; unpaired $t$ test, $t_{(30)}=0.7262, p=0.4733$; Fig. $\left.10 B\right]$. Moreover, the ratio between Or42a OSN FMRP and total FMRP (i.e., FMRP in all maxillary palp OSNs) is not different between controls and TeTxLc animals [control $1.151 \pm 0.03487(n=20)$ vs Or42a-Gal4>TeTxLc $1.176 \pm 0.2347(n=12)$; unpaired $t$ test, $t_{(30)}=0.3627, p=0.7193$; Fig. 10C]. Together, these results suggest silencing Or42a OSN synaptic output does not detectably alter FMRP levels, again implicating the importance of lateral connections from other neurons rather than a direct feedback mechanism.

\section{Critical period olfactory experience selectively remodels presynaptic OSN innervation}

Previous work from our lab and others has shown that critical period odorant experience can drive dendritic arbor changes in postsynaptic PNs downstream of OSN glomerular innervation (Sachse et al., 2007; Doll and Broadie, 2015; Chodankar et al., 2020). However, a recent study has suggested that Or42a OSN presynaptic remodeling is a completely separable mechanism (Chodankar et al., 2020). This recent study, combined with Or42a OSN remodeling in absence of synaptic output to VM7 PNs, made it unclear whether presynaptic innervation changes would be mirrored in a comparable PN postsynaptic refinement. 
We therefore next jointly assayed both the presynaptic OSNs and the postsynaptic PNs following the critical period odorant exposure. To simultaneously image presynaptic and postsynaptic partners within the VM7 glomerulus, we labeled the Or42a OSNs with Or42a-mCD8::4xGFP (Fig. 11A, left, green), while using NP3481-Gal4 (Olsen and Wilson, 2008) to drive UAS-mCD8::RFP (NP3481>mCD8::RFP) in the PNs (Fig. 11A, middle, magenta). The co-labeling shows the RFPmarked PNs overlap with the GFP-marked Or42a OSNs within the VM7 glomerulus in merged single slice confocal images (Fig. 11A, right, merged). These animals were exposed to either oil vehicle or $20 \%$ EB during the 0 - to 2-dpe critical period. Representative images of the presynaptic and postsynaptic processes in the VM7 glomerulus, and the innervation quantifications for both Or42a OSNs and VM7 PNs following critical period treatments, are shown in Figure 11.

Following 20\% EB critical period exposure, the control animals show the typical strong reduction of Or42a OSN innervation (Fig. 11B, green, left vs right). Despite loss of presynaptic innervation, postsynaptic PNs are largely unchanged between oil-exposed and EB-exposed conditions (Fig. 11B, magenta, left vs right). Presynaptic and postsynaptic volumes were quantified and compared with $t$ tests with Sidak's correction and a simple linear regression. Compared with oil-exposed animals, EB-exposed animals show significantly reduced Or42a OSN innervation, but no significant difference in the PN volume in the VM7 glomerulus [Or42a OSN oil $1.0 \pm 0.07 \quad(n=18)$ vs $20 \%$ EB $0.15 \pm 0.06$ $(n=18) ; t_{(34)}=9.43, p=1.04 \mathrm{e}-10 ; \mathrm{VM} 7 \mathrm{PN}$ oil $1.24 \pm 0.04$ $(n=18)$ vs $20 \%$ EB $1.1 \pm 0.11(n=18)$ PN volumes normalized to Or42a OSN oil volume mean; $t_{(34)}=1.12, p=0.47$; Fig. $11 C$ ]. Note that there is some increased variability in the VM7 PN volumes in the EB-exposed animals compared with vehicle controls (SEM oil 0.04 vs 20\% EB 0.11; Fig. 11C). We therefore tested whether Or42a OSN innervation and PN dendritic arborization might correlate, and possibly account for some of the odorantinduced variability. A simple linear regression was used to compare the presynaptic and postsynaptic volumes, but only a very weak, EB-independent relationship is apparent (oil $R^{2}=0.1591$ vs EB $R^{2}=0.1387$; Fig. $11 D$ ). In summary, these results show that critical period odorant exposure drives striking remodeling in the presynaptic Or42a OSNs, but not the postsynaptic PNs, and therefore separates this process from similar processes that have mirrored presynaptic and postsynaptic alterations.

\section{Silencing AL glutamatergic interneurons reduces Or42a OSN critical period remodeling}

AL LNs are prime candidates to regulate critical period remodeling. LNs interconnect OSNs for lateral modulation of OSN activity (Chou et al., 2010; Fig. 8) receive broad synaptic output from many OSNs (Olsen and Wilson, 2008; Fig. 9), and innervate OSN presynaptic terminals (Wilson, 2013; Fig. 11). LNs release neuromodulators including GABA and glutamate (Jackson et al., 1990; Das et al., 2010). We previously found that Or42a-targeted knock-down of the glutamate receptor NMDAR1 subunit strongly impairs Or42a OSN critical period remodeling (Golovin et al., 2019). Glutamatergic LNs (GluLNs; Fig. 1E, bottom right) provide the major source of glutamate neurotransmission in the AL circuit (Liu and Wilson, 2013). Therefore, we next tested the contribution of the GluLNs to Or42a OSN critical period remodeling. GluLN synaptic output was silenced as above, using OK107-Gal4 to drive tetanus toxin (OK107-Gal4>TeTxLc), with Or42a OSNs labeled by Or42a-mCD8::4xGFP. Since the OK107-Gal4 driver has extensive expression in the MB (Connolly et al., 1996), we also used the MB-restricted driver MB247-Gal4 (Zars et al., 2000) as a control to express TeTxLc (MB-247-Gal4>TeTxLc) and assess the effect of MB silencing on Or42a critical period remodeling. The same transgenic lines lacking TeTxLc were used as controls. Staged animals from all genotypes were exposed to either the oil vehicle or $20 \%$ EB during the 0 - to 2-dpe critical period. Representative VM7 images and glomerulus innervation quantifications for all genotypes and conditions are shown in Figure 12.

As above, transgenic control animals show a striking reduction in VM7 glomerulus innervation with $20 \% \mathrm{~EB}$ critical period exposure (Fig. 12A, top). In contrast, animals with GluLN silencing by TeTxLc (OK107-Gal4>TeTxLc) have impaired Or42a OSN remodeling following the EB odorant exposure (Fig. 12A, top vs bottom). Quantification of glomerulus innervation and ANOVA $(2 \times 2)$ analyses show a significant effect of the odor $\left(F_{(1,74)}=367.9, p=1.946 \mathrm{e}-30\right)$ and the GluLN TeTxLc silencing $\left(F_{(1,74)}=10.24, p=0.002\right)$, with a significant interaction between experience and genotype $\left(F_{(1,74)}=25.61, p=2.95 \mathrm{e}-6\right.$; Fig. $\left.12 B\right)$. Pairwise comparisons using the $t$ tests with Sidak's corrections show that EB-exposed animals have significantly reduced innervation compared with oil-exposed transgenic controls and OK107-Gal4>TeTxLc animals [control oil $1.0 \pm 0.03(n=26)$ vs $20 \%$ EB $0.003 \pm 0.001 \quad(n=25) ; t_{(74)}=20.61, p=1.31 \mathrm{e}-31$; 


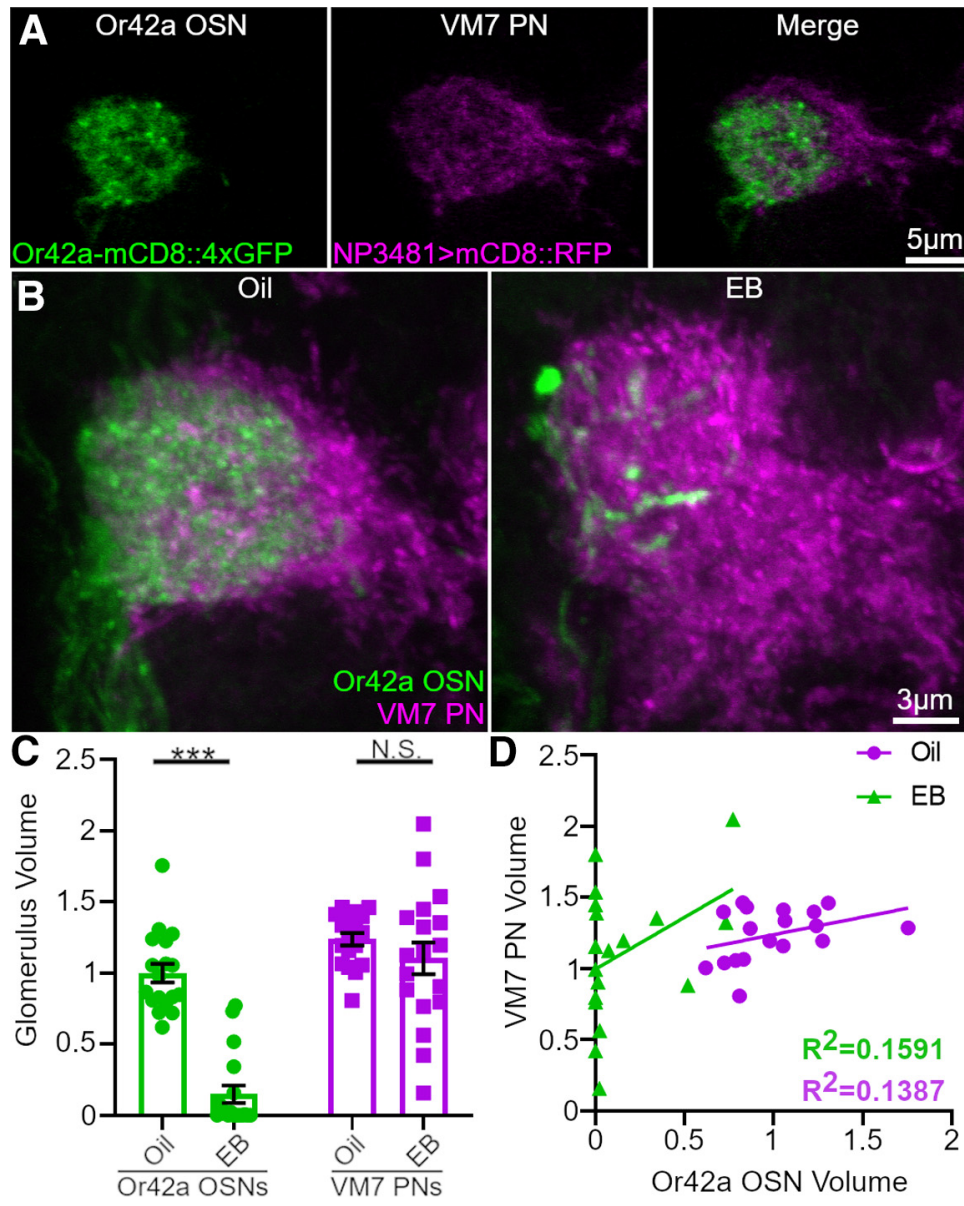

Figure 11. Odorant exposure selectively remodels presynaptic OSNs in the VM7 glomerulus. $A$, Representative confocal slices showing presynaptic Or42a 0SNs (Or42a-mCD8::4xGFP, green; left) and postsynaptic PNs (NP3481-Gal4>mCD8::RFP, magenta; middle), with the merged image (right). $\boldsymbol{B}$, Representative VM7 merged images after exposure to the oil vehicle alone (left) or 20\% EB odorant (right) during the 0 - to 2-dpe critical period. C, Quantification of VM7 glomerulus volume of Or42a OSNs (green) and VM7 PNs (magenta), normalized to the vehicle control. Data shown as a scatter plot of all data points with mean \pm SEM. $\boldsymbol{D}$, Quantification of the relationship between the presynaptic Or42a OSN volume and postsynaptic PN volume within the VM7 glomerulus. Data shown as a scatter plot with lines fit to vehicle (magenta) and EB (green) conditions. $R^{2}$ values given for each condition. Significance is presented as not significant (N.S; $p>0.05$ ), and significant at $* * * p<0.001$

TeTxLc oil $0.923 \pm 0.06 \quad(n=13) \quad$ vs $20 \%$ EB $0.343 \pm 0.07$ $(n=14) ; t_{(74)}=8.73, p=3.23 \mathrm{e}-12$; Fig. $10 B$, bottom bars]. Silencing GluLNs does not alter the basal glomerulus innervation under control oil-exposed conditions (control oil vs TeTxLc oil; $t_{(74)}=1.304, p=0.7303$ ), but does significantly increase Or42a OSN innervation following the critical period EB odorant exposure (control $20 \% \mathrm{~EB}$ vs TeTxLc $20 \% \mathrm{~EB} ; t_{(74)}=5.893, p=6.27 \mathrm{e}-$ 7; Fig. 12B, top bar). Together, these results suggest a role for GluLN glutamatergic signaling in Or42a OSN olfactory-experience-dependent critical period remodeling, but it is possible that some or all of this effect is because of OK107-Gal4 expression within the downstream MB learning/memory center.

MB-restricted silencing with MB247-Gal4 slightly enhances the Or42a OSN remodeling (Fig. 12C). Like transgenic controls, MB247-Gal4>TeTxLc animals exposed to $20 \%$ EB show reduced VM7 innervation, but the effect is even stronger than in the controls. Quantification of innervation and ANOVA $(2 \times 2)$ analyses show a significant effect of both odor $\left(F_{(1,64)}=136.9, p=1.513 \mathrm{e}-17\right)$ and genotype $\left(F_{(1,64)}=4.459, p=0.0386\right)$, but no significant interaction between them $\left(F_{(1,64)}=0.743, p=0.3919\right.$; Fig. 12D). Pairwise comparisons using $t$ tests with Sidak's corrections show that EB-exposed animals have significantly reduced innervation compared with both oilexposed transgenic controls and MB247Gal4 $>$ TeTxLc animals [control oil $1.0 \pm 0.03$ $(n=25)$ vs $20 \%$ EB $0.357 \pm 0.07(n=20) ; t_{(64)}=$ 9.287, $p=1.07 \mathrm{e}-12$; TeTxLc oil $0.926 \pm 0.07(n=12)$ vs $20 \%$ EB $0.181 \pm 0.08 \quad(n=11) ; t_{(64)}=7.736$, $p=5.65 \mathrm{e}-10$; Fig. $12 D$, bottom bars]. Silencing the $\mathrm{MB}$ does not significantly alter basal glomerulus innervation under oil-exposed control conditions (control oil vs TeTxLc oil; $t_{(64)}=0.9146, p=$ 0.3639 ), but does significantly decrease Or42a OSN innervation following critical period EB exposure (control $20 \%$ EB vs TeTxLc $20 \%$ EB; $t_{(64)}$ $=2.036, p=0.0459$; Fig. $12 D$, top bar). Despite the significant decrease in innervation volume in the MB247>TeTxLc EB condition compared with the transgenic control, the lack of a significant interaction term between genotype and odorant exposure in the ANOVA complicates interpretation. Taken together, these results indicate GluLNs role in critical period OSN remodeling, but do not clearly demonstrate a potential $\mathrm{MB}$ role.

Based on the above findings, we hypothesized that GluLN-released glutamate binds to Or42a OSN NMDARs to modulate critical period remodeling. To further test this hypothesis, we next set forth to assess whether NMDAR1 specifically regulates critical period remodeling. Transgenic controls with Or42a OSNs labeled using Or42a-mCD8::GFP were compared in two Nmdar1 mutant combinations: (1) Nmdar1 null mutants homozygous for a $\mathrm{Mi}\{\mathrm{MIC}\}$ insertion

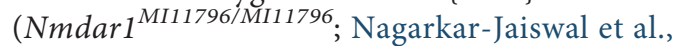
2015); and (2) heterozygous mutants with a copy of Nmdar1 $1^{\text {MI11796 }}$ over a hypomorphic mutation (Nmdar1 ${ }^{\text {EP331/MI11796; Rorth, 1996; }}$ Xia et al., 2005). Control and mutant animals were exposed to either oil vehicle or $20 \%$ EB in the 0 - to 2-dpe critical period. Representative images of the Or42a OSN VM7 glomerular innervation, as well as innervation quantifications and the effect of EB exposure for all genotypes and conditions, are all shown in Figure 13. Transgenic control animals show the characteristic strong reduction in VM7 innervation following $20 \%$ EB critical period exposure (Fig. $13 \mathrm{~A}$, left). Surprisingly, we found that both mutants also show a very similar response to the EB exposure. Mutants exhibit a strong reduction in Or42a OSN axon terminal innervation and the characteristic OSN bright puncta (Fig. 13A, middle and right). This suggests two possibilities: (1) the effect of GluLNs silencing on Or42a OSN critical period remodeling does not rely on NMDAR1-dependent signaling; or (2) Or42a-targeted NMDAR1 knock-down may have a similar effect as with FMRP with only circuit imbalance having an impact on critical period remodeling.

ANOVA $(3 \times 2)$ quantification shows a significant effect of both odorant $\left(F_{(1,128)}=567.7, p=6.876 \mathrm{e}-49\right)$ and genotype $\left(F_{(2,128)}=\right.$ 6.084, $p=0.003)$, but no significant interaction between them $\left(F_{(2,128)}=2.089, p=0.128\right.$; Fig. $\left.13 B\right)$. Pairwise comparisons using Sidak's corrected $t$ tests show that both control and mutants 
significantly reduce innervation volumes with critical period EB exposure [control oil $1.0 \pm 0.03 \quad(n=32) \quad$ vs $20 \% \quad$ EB $0.085 \pm$ $0.03(n=33) ; t_{(128)}=17.73, p=4.25 \mathrm{e}-35$; Nmdar1 ${ }^{\text {MI11796/MI11796 }}$ oil $0.873 \pm 0.06(n=13)$ vs $20 \%$ EB $0.078 \pm 0.03(n=18) ; t_{(128)}=10.5$, $p=8.29 \mathrm{e}-18 ;$ Nmdar1 $^{E P 331 / M I 11796}$ oil $1.149 \pm$ $0.08(n=19)$ vs $20 \%$ EB $0.147 \pm 0.05(n=19)$; $t_{(128)}=14.85, p=1.9 \mathrm{e}-28$; Fig. $13 B$, bottom bars], but there is no significant difference in the basal innervation with vehicle exposure

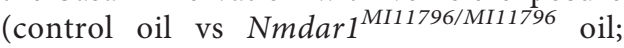
$t_{(128)}=1.858, p=0.6376$; control oil vs

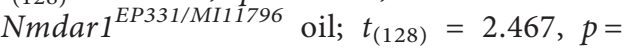
$0.202)$ or in odor-dependent remodeling (control EB vs Nmdar1 ${ }^{\text {MI11796/MI11796 EB; }}$ $t_{(128)}=0.113, p>0.9999 ;$ control EB vs Nmdar1 ${ }^{\text {EP331/MI11796 }} \mathrm{EB} ; t_{(128)}=1.029$, $p=0.9958$; Fig. $13 B$, middle and top bars). Quantification of EB effects using multiple linear regression shows that all genotypes have similar responses without significantly different regression coefficients $(20 \%$ EB x Nmdar1 ${ }^{\text {MI11796/MI11796 }} \beta=0.1203 \pm 0.09 ; t_{(128)}=$ $1.312, p=0.1918 ; 20 \%$ EB x Nmdar1 $1^{\text {EP331/MI11796 }}$ $\beta=-0.08,699 \pm 0.08 ; t_{(128)}=1.024, p=0.3079$; Fig. 13C). Together, these results suggest that NMDAR1 signaling functions in an Or42a OSNspecific pattern similar to FMRP to mediate the olfactory experience-dependent critical period remodeling of Or42a OSN presynaptic terminals.

\section{Or42a OSN-targeted GABA $\mathrm{A}$ knock-down enhances critical period innervation remodeling}

Inhibitory GABAergic LNs oppose excitatory inputs, with the excitatory and inhibitory LN balance controlling AL circuit output (Acebes et al., 2011, 2012). Excitatory/inhibitory imbalance characterizes FXS disease models (Contractor et al., 2015). Therefore, we hypothesized altering inhibitory LN signaling might imbalance the AL circuit and impair Or42a OSN refinement. We showed above that remodeling depends on neural activity specifically within the Or42a OSNs. In addition, we showed that Or42a OSN critical period remodeling is augmented when other OSNs cannot transduce odorants. Since the overall impact of OSN population interactions on Or42a OSNs is lateral inhibition (Olsen and Wilson, 2008), we hypothesized that reducing inhibition should mimic the effects of the orco mutant with Or42a-Gal4 $>$ Orco rescue (Fig. 7 ). Since a major component of lateral inhibition onto Or42a OSNs comes from ionotropic GABA receptors (Olsen and Wilson, 2008), we used Or42a-targeted RNAi to knock-down resistant to dieldrin (RDL), a principle $\mathrm{GABA}_{\mathrm{A}} \mathrm{R}$ subunit (Aronstein and Ffrench-Constant, 1995; Okada et al., 2009). In order to examine the role of RDL in the critical period remodeling of Or42a OSNs, we used Or42a-Gal4>mCD8::GFP to label the neurons and compared the RDL RNAi expressing animals (Or42a-Gal4 $>$ Rdl RNAi) to a transgenic control expressing only the membrane-bound GFP. Staged animals were exposed
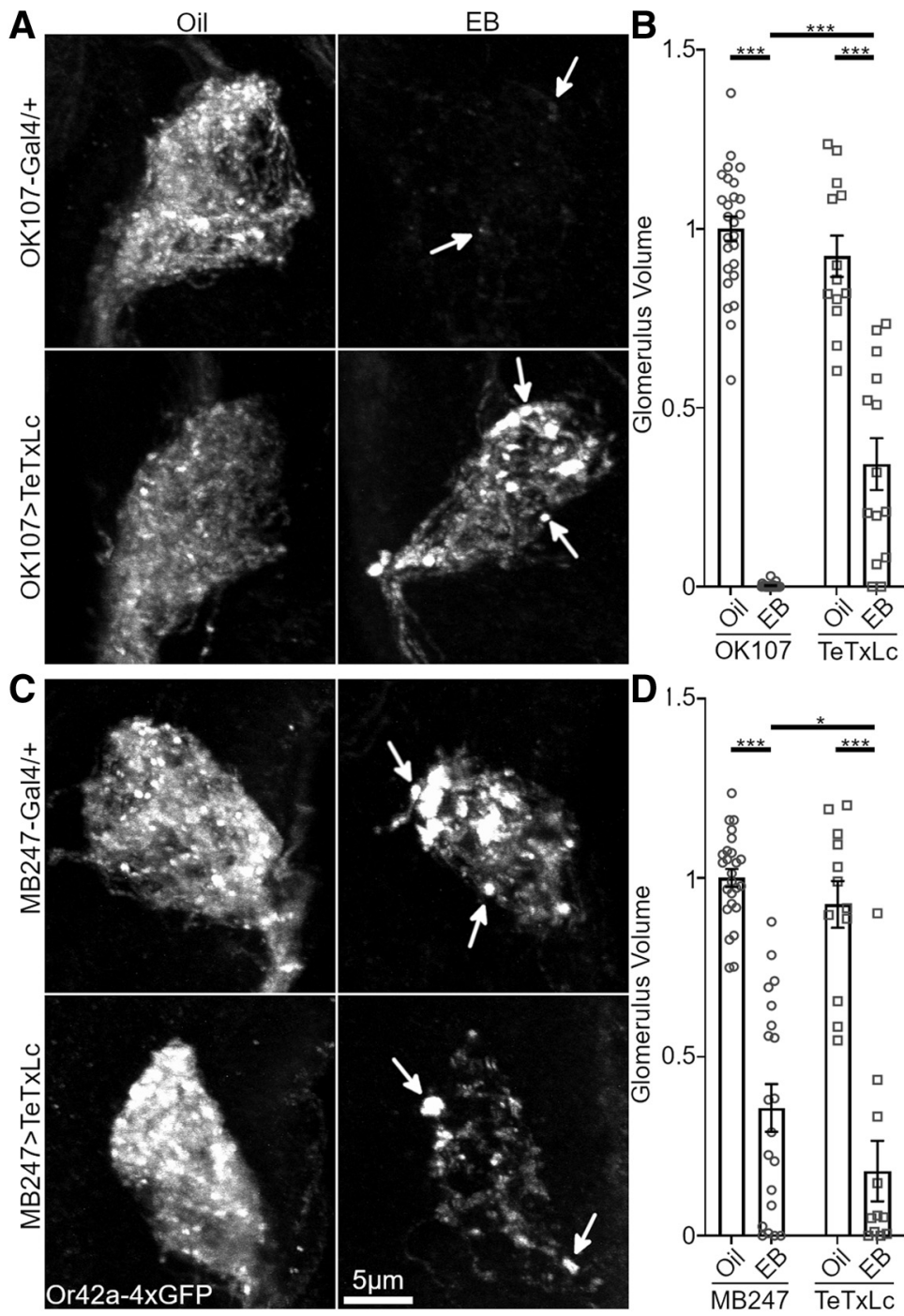

Figure 12. Silencing AL glutamatergic neurons reduces 0r42a OSN critical period remodeling. $\boldsymbol{A}_{t}$ Representative confocal maximum intensity projections of 0r42a OSN VM7 innervation (two copies of ther oil or EB. C, Imaging as above in $\boldsymbol{A}$, transgenic control ( $w^{-} / w^{-} ;$Or42a-mCD8::4xGFP/0r42amCD8::4xGFP; MB247-Gal4/+; top) and with MB247-Gal4 driving UAS-TeTxLc (bottom). Females exposed Gal4 control and TeTxLc animals exposed to oil or EB. Scatter plots show all data points and the mean \pm SEM. Significance is indicated as $* p<0.05$ and $* * * p<0.001$

to either oil vehicle or $20 \% \mathrm{~EB}$ during the 0 - to 2-dpe critical period. Representative images of Or42a OSN innervation of the VM7 glomerulus as well as innervation quantifications for all conditions are shown in Figure 14.

As in all studies above, control animals EB-exposed during the critical period exhibit a strong reduction in Or42a OSN innervation of the VM7 glomerulus (Fig. 14A, top). When $\mathrm{GABA}_{\mathrm{A}}$ signaling is reduced with Or42a OSN-targeted $R d l$ RNAi, basal innervation is similar to controls following oil exposure (Fig. 14A, left, top vs bottom). However, when Or42aGal4 $>$ Rdl RNAi animals are exposed to EB during the critical period, they have a larger reduction in Or42a OSN innervation compared with controls (Fig. 14A, right, top vs bottom). ANOVA $(2 \times 2)$ quantification shows significant effects of odor 


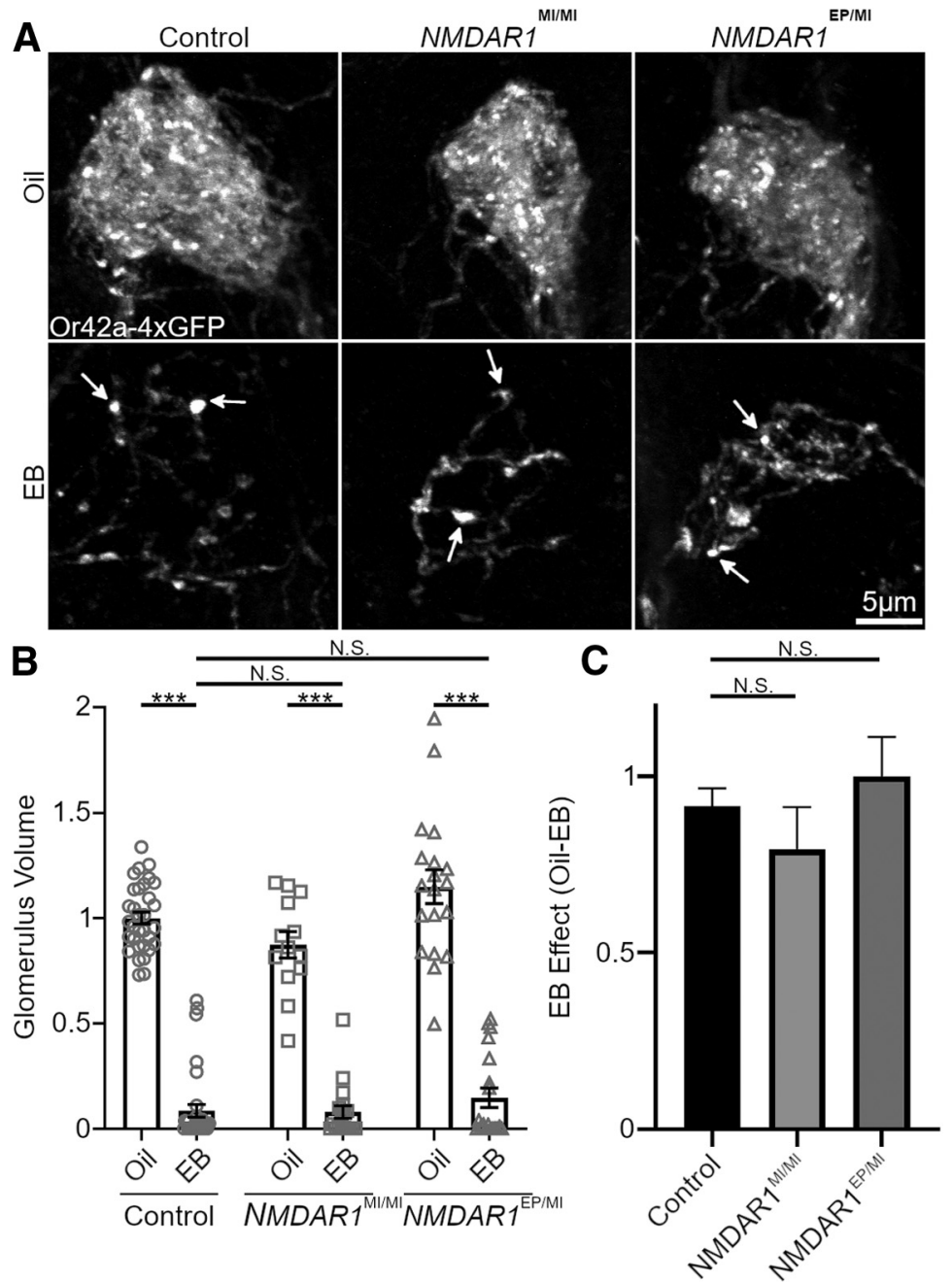

Figure 13. NMDAR1 signaling is not required for OSN critical period innervation remodeling. $\boldsymbol{A}$, Representative confocal maximum intensity projections of Or42a OSN VM7 innervation (two copies of Or42amCD8::4xGFP; white) following exposure to oil vehicle (top) or 20\% EB (bottom) during the 0- to 2-dpe critical period. Three genotypes are shown: transgenic control ( $w$; Or42a-mCD8::4xGFP/Or42a-mCD8::4xGFP; left), NMDAR1 mutant (NMDAR1 ${ }^{\text {MI11796; }}$; middle) and a second NMDAR1 mutant (NMDAR1 EP331/NMDAR1 $1^{\text {Ml11796; }}$; right). Remnant puncta following EB exposure are labeled by white arrows. $B$, Quantification of VM7 innervation volume for each genotype and condition. $\boldsymbol{C}$, The difference between oil and EB conditions for each genotype. Scatter plots show all data points and the mean \pm SEM. Bar graphs show mean \pm SER. Significance is indicated as not significant (N.S.; $p>0.05$ ) and $* * * p>0.001$.

$\left(F_{(1,98)}=259.6, p=2.671 \mathrm{e}-29\right)$ and $\operatorname{Rdl} \operatorname{RNAi}\left(F_{(1,98)}=19.78\right.$, $p=2.3 \mathrm{e}-5$; with a significant interaction between the two $\left(F_{(1,98)}\right.$ $=17.11, p=7.5 \mathrm{e}-5)$; Fig. 14B]. Pairwise comparisons with $t$ tests and Sidak's correction show both control and Rdl RNAi animals have significantly reduced Or42a OSN innervation after EB exposure [control oil $1.0 \pm 0.03 \quad(n=26)$ vs $20 \%$ EB $0.538 \pm 0.04(n=28) ; t_{(98)}=8.729, p=4.14 \mathrm{e}-13 ; R d l$ RNAi oil $0.988 \pm 0.04(n=23)$ vs $20 \%$ EB $0.206 \pm 0.04(n=25) ; t_{(98)}=$ $14.57, p=1.73 \mathrm{e}-25$; Fig. $14 B$, bottom bars]. Although the two genotypes had comparable innervation with vehicle (control oil vs $R d l$ RNAi oil; $\left.t_{(98)}=0.2161, p>0.9999\right)$, Or $42 a-G a l 4>R d l$ RNAi significantly decreases the VM7 glomerulus innervation following critical period EB exposure (control EB vs $R d l$ RNAi $\mathrm{EB} ; t_{(98)}=6.193, p=8.32 \mathrm{e}-8$; Fig. $14 B$, top bar). These results reveal a role of $\mathrm{GABA}_{\mathrm{A}} \mathrm{R}$-mediated inhibition in regulating Or42a OSN synaptic remodeling, and provide a mechanism by which FMRP acts to modulate olfactory experience-dependent critical period refinement.

\section{Discussion}

The first days of Drosophila adulthood mark a critical period for the remodeling of brain olfactory circuitry (Devaud et al., 2003; Sachse et al., 2007; Tessier and Broadie, 2009; Doll and Broadie, 2015; Golovin et al., 2019; Chodankar et al., 2020). During this developmental window, OSNs manifest heightened adaptability to the new odorant sensory environment. Vertebrates show similarly heightened responsiveness to early odorant exposure. Rodents exposed to odors during development show increased or decreased effects dependent on conditions examined (Dalland and Døving, 1981; Geramita and Urban, 2016; Liu and Urban, 2017). Zebrafish also exhibit olfactory imprinting from developmental odorant exposure (Gerlach et al., 2019). We previously described OSN innervation remodeling that is restricted to an early-life critical period (02 dpe; Golovin et al., 2019). Here, we expand our knowledge of this remodeling by testing the role of FMRP, a protein strongly implicated in activity-dependent critical periods (Dölen et al., 2007; Tessier and Broadie, 2009; Contractor et al., 2015). Based on this study, we propose that FMRP functions to regulate the lateral interactions between OSNs mediated by LNs (Acebes et al., 2011). Previous studies have shown that each OSN receives lateral presynaptic GABAergic inhibition that scales with the total activity of all OSNs, and serves as a gain control mechanism by reducing OSN activity to odorants that activate multiple OSN classes (Olsen and Wilson, 2008). High EB concentrations likely activate multiple OSN classes, in addition to the Or42a OSNs, and should therefore recruit presynaptic inhibition. We would expect this inhibition to limit activity in response to EB exposure and reduce remodeling. Indeed, when we block lateral inhibition either through (1) specifically activating Or42a OSNs or (2) selectively removing Or42a OSN $\mathrm{GABA}_{\mathrm{A}}$ receptors, EB odorant exposure has a greater effect on critical period remodeling.

In addition, our previous work showed that Or42a-targeted NMDAR1 knock-down impairs Or42a OSN innervation remodeling, likely via reduced lateral excitation (Golovin et al., 2019). However, NMDAR signaling on OSNs has not been directly shown. Or42a-targeted FMRP RNAi impairs the critical period remodeling of Or42a OSNs. Or42aspecific FMRP loss likely alters the OSN response to circuit lateral inputs both by enhancing inhibition and also reducing excitation. This role represents a novel FMRP function for regulating acute OSN remodeling specifically on the presynaptic side (Sudhakaran et al., 2014; Doll and Broadie, 2015, 2016; Franco et al., 2017). Previous studies have found that FMRP is required for mediating long-term habituation (LTH), a form of structural and functional adaption that leads to a reduction in innate avoidance behavior (Das et al., 2011). More specifically, FMRP interacts with a second RNA-binding protein (Ataxin2), contributing to LTH by acting in both the PNs and LNs (Sudhakaran et al., 2014). FMRP-dependent PN remodeling likewise occurs following a single day of activation within a cell-autonomous 


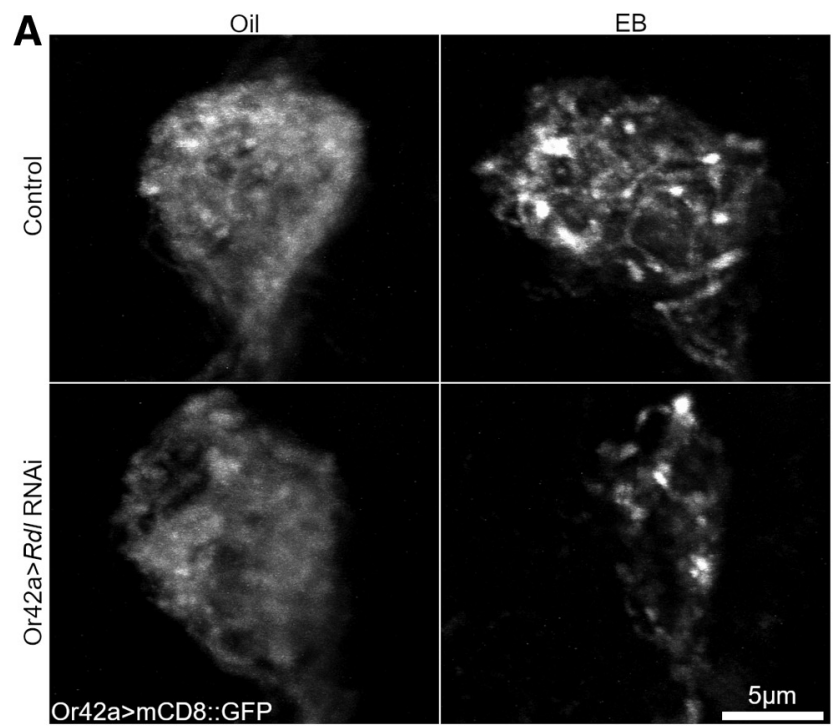

B

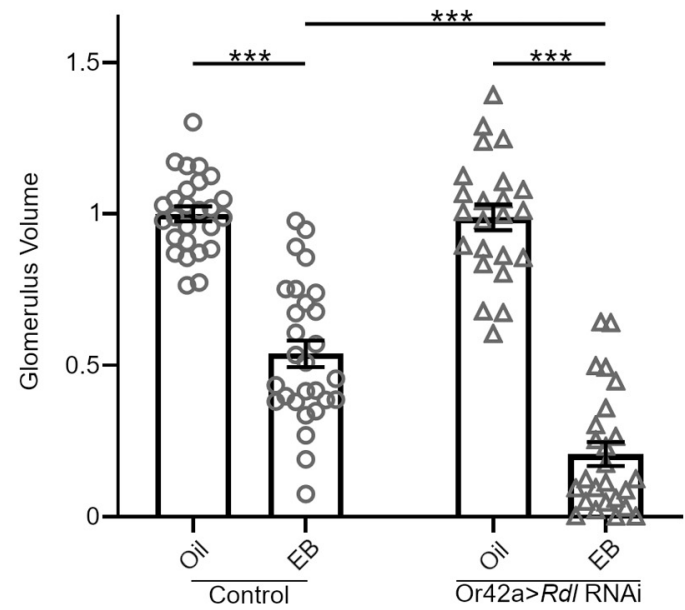

Figure 14. 0 r42a OSN-targeted $G A B A_{A} R$ knock-down enhances critical period remodeling. $A$, Representative confocal maximum intensity projections of 0r42a OSN VM7 innervation (Or42a-Gal4>UAS-mCD8::GFP; white) following exposure to oil vehicle (top) or 20\% EB odorant (bottom) during the 0 - to 2-dpe critical period. Two genotypes are shown; transgenic control (Or42a-Gal4>mCD8::GFP; top) and Or42a-targeted Rdl RNAi (Rdl RNAi 8-10J; bottom). $\boldsymbol{B}$, Quantification of VM7 innervation for the two genotypes and conditions. Scatter plots show all data points with the mean \pm SEM. The significance is indicated as $* * * p<0.001$.

mechanism (Doll and Broadie, 2015, 2016). The results presented here extend beyond this earlier work in three ways: (1) Or42a OSN remodeling involves lateral inhibition mechanisms; (2) Or42a OSN remodeling is a purely presynaptic process; and (3) Or42a OSN remodeling manifests acute reversibility (Chodankar et al., 2020). The neuron-specific FMRP functions in the AL olfactory circuit highlight an increasingly appreciated FMRP role specificity within different neuron classes.

FMRP is widely expressed in the nervous system (Khandjian et al., 1995; Zhang et al., 2001), where it binds multiple different target mRNAs, including neuron class-specific transcripts (Darnell et al., 2011). For example, a recent study in mice showed that FMRP binds to circadian protein-encoding mRNAs preferentially in hippocampal CA1 pyramidal neurons compared with cerebellar granule neurons (Sawicka et al., 2019). Therefore, it is important to identify how FMRP loss perturbs the function of specific neuron classes to affect particular neural circuits. The current study describes how unbalancing FMRP levels between neurons of the Drosophila olfactory circuit can alter odorant experience-dependent remodeling, revealing how dissecting FMRP functions at a fine cellular resolution can uncover FXS circuit-level impairments (Contractor et al., 2015; Franco et al., 2017; Goel et al., 2018; Lovelace et al., 2020). Neuron class-specific FMRP functions are also revealed by activity-dependent dissection, with optogenetic stimulation causing opposite phenotypes in the olfactory PN and MB output neurons, but both effects failing when FMRP is absent (Doll and Broadie, 2015). Functionally, recent work on the FMRP role in binding to HCN cation channels demonstrates opposing excitability consequences in hippocampal CA1 compared with layer 5 prefrontal cortex (Brandalise et al., 2020). Together with the results presented here, this work underscores the importance of neuron-specific FMRP mechanisms, and the need to understand how these altered roles combine to generate circuit-level FXS phenotypes.

One intriguing result from our experiments is that despite the important FMRP role in Or42a remodeling, FMRP null mutants maintain normal remodeling capacity. We suggest that this compensation is because of AL LN circuitry that allows for OSN activity to be modified based on the total input to the system (Olsen and Wilson, 2008). LNs excitation scales with the total OSN activity, so when FMRP is altered (LOF/GOF) only in Or42a OSNs there is little change to LN output. However, when FMRP is manipulated in all OSNs equally, the output of LNs is adjusted based on the responsiveness of all the OSNs, thereby balancing the circuit (Olsen et al., 2010; Mohamed et al., 2019). Although this result was unexpected by us, it is not unprecedented for homeostatic mechanisms to be able to overcome FMRP loss (Antoine et al., 2019; Domanski et al., 2019). For instance, in a recent analysis of four monogenic mouse autism models, including FXS, there was an increase in the excitatoryto-inhibitory ratio of pyramidal neurons within the primary somatosensory cortex, which in general served as a homeostatic mechanism to maintain the overall network activity, but there was not increased excitatory spiking (Antoine et al., 2019). Another example from mouse barrel cortex demonstrates that although the development of NMDA-dependent LTP is disrupted in the FXS disease model, there is no apparent defect in lesion-induced plasticity owing to homeostatic compensation (Harlow et al., 2010). In order to more fully understand the FXS condition, we must grasp not only neuron-specific FMRP functions, but also how these functions balance across circuits.

Our results indicate the balanced roles of inhibitory $\mathrm{GABA}_{\mathrm{A}} \mathrm{R}$ and excitatory NMDAR signaling in OSN remodeling. In our previous report on critical period remodeling, we found that Or42a-targeted NMDAR1 RNAi impairs the innervation loss from early-life EB odorant exposure (Golovin et al., 2019). Here, we find that targeted tetanus toxin synaptic silencing of GluLNs, the major source of AL glutamatergic transmission (Das et al., 2010), causes a similar impairment to Or42a OSN-targeted NMDAR1 knock-down. However, global Nmdar1 mutants lack a detectable phenotype. One explanation is that NMDAR signaling acts in a similar fashion to FMRP function, with targeted removal putting Or42a OSNs out of balance with the rest of the circuit, but global loss not generating this imbalance. Since the AL has both metabotropic glutamate receptors (Devaud et al., 2008) and glutamate-gated chloride channels (Liu and Wilson, 2013), GluLNs could mediate their effect on Or42a remodeling by also altering signaling through these receptors. Another unlikely possibility is that since the OK107-Gal4 driver expresses in a few neurons outside the olfactory circuitry (Aso et al., 2009) these distant populations might be mediating the effects. Future 
experiments testing broader NMDAR1 functions, as well as studies of possible mGLuR-mediated glutamate signaling in AL, will be important to fully elucidate circuit mechanisms that regulate OSN remodeling.

In conclusion, we discover here that unbalanced neuron class-specific FMRP functions can alter lateral OSN interactions and impact critical period OSN remodeling. The findings show that FMRP acts in Or42a OSNs as well as other EB-responsive OSNs to control the response to lateral input. When FMRP is removed only in Or42a OSNs, they have a lower responsiveness to $\mathrm{EB}$ exposure and therefore manifest impaired critical period remodeling. However, when FMRP is removed from all OSNs, balance is restored to reinstate the normal remodeling. The FMRP requirement is bidirectional as targeted FMRP elevation in Or42a OSNs also causes circuit imbalance to enhance the effect of odorant experience on critical period remodeling. Furthermore, unlike other forms of activity-dependent remodeling in the $\mathrm{AL}$ circuit, this reversible remodeling occurs only in the presynaptic OSN terminals and requires OSN activity, but not OSN synaptic output. Or42a OSNtargeted optogenetic activation drives critical period remodeling, but this activity-dependent mechanism does not require FMRP function in the Or42a OSNs. Despite the clear involvement of LN glutamatergic and GABAergic signaling in regulating OSN critical period remodeling, their exact circuit connectivity remains to be fully elucidated. The innervation loss and retraction characteristics following critical period odorant experience resemble developmental pruning during Drosophila metamorphosis, which suggests similar underlying mechanisms of cytoskeleton disassembly and glial phagocytosis ( $\mathrm{Yu}$ and Schuldiner, 2014). In addition, the mechanism(s) by which FMRP regulates OSN responses to lateral inputs will be an important avenue for future research. Overall, this work provides a new example of neuron class-specific FMRP function, neural circuit compensation for FMRP loss, and an avenue to inform therapies addressing FXS circuit-level symptoms.

\section{References}

Acebes A, Martín-Peña A, Chevalier V, Ferrús A (2011) Synapse loss in olfactory local interneurons modifies perception. J Neurosci 31:2734-2745.

Acebes A, Devaud J, Arnés M, Ferrús A (2012) Central adaptation to odorants depends on PI3K levels in local interneurons of the antennal lobe. J Neurosci 32:417-422.

Antoine M, Langberg T, Schnepel P, Feldman D (2019) Increased excitationinhibition ratio stabilizes synapse and circuit excitability in four autism mouse models. Neuron 101:648-661.e4.

Aronstein K, Ffrench-Constant R (1995) Immunocytochemistry of a novel GABA receptor subunit Rdl in Drosophila melanogaster. Invert Neurosci 1:25-31.

Aso Y, Grübel K, Busch S, Friedrich A, Siwanowicz I, Tanimoto H (2009) The mushroom body of adult Drosophila characterized by GAL4 drivers. J Neurogenet 23:156-172.

Bailey D, Hatton D, Skinner M (1998) Early developmental trajectories of males with fragile X syndrome. Am J Mental Retard 103:29.

Bolduc F, Bell K, Cox H, Broadie K, Tully T (2008) Excess protein synthesis in Drosophila Fragile X mutants impairs long-term memory. Nat Neurosci 11:1143-1145.

Brandalise F, Kalmbach B, Mehta P, Thornton O, Johnston D, Zemelman B, Brager D (2020) Fragile X mental retardation protein bidirectionally controls dendritic Ih in a cell-type specific manner between mouse hippocampus and prefrontal cortex. J Neurosci 40:5327-5340.

Brown V, Jin P, Ceman S, Darnell J, O’Donnell W, Tenenbaum S, Jin X, Feng Y, Wilkinson K, Keene J, Darnell R, Warren S (2001) Microarray identification of FMRP-associated brain mRNAs and altered mRNA translational profiles in fragile $\mathrm{X}$ syndrome. Cell 107:477-487.

Bureau I, Shepherd G, Svoboda K (2008) Circuit and plasticity defects in the developing somatosensory cortex of Fmr1 knock-out mice. J Neurosci 28:5178-5188.

Chiang A, Priya R, Ramaswami M, VijayRaghavan K, Rodrigues V (2009) Neuronal activity and Wnt signaling act through Gsk3- $\beta$ to regulate axonal integrity in mature Drosophila olfactory sensory neurons. Development 136:1273-1282.

Chodankar A, Sadanandappa M, VijayRaghavan K, Ramaswami M (2020) Glomerulus-selective regulation of a critical period for interneuron plasticity in the Drosophila antennal lobe. J Neurosci 40:5549-5560.

Chou Y, Spletter M, Yaksi E, Leong J, Wilson R, Luo L (2010) Diversity and wiring variability of olfactory local interneurons in the Drosophila antennal lobe. Nat Neurosci 13:439-449.

Contractor A, Klyachko V, Portera-Cailliau C (2015) Altered neuronal and circuit excitability in fragile X syndrome. Neuron 87:699715 .

Connolly J, Roberts I, Armstrong J, Kaiser K, Forte M, Tully T, O'Kane C (1996) Associative learning disrupted by impaired Gs signaling in Drosophila mushroom bodies. Science 274:21042107.

Couto A, Alenius M, Dickson B (2005) Molecular, anatomical, and functional organization of the Drosophila olfactory system. Curr Biol 15:1535-1547.

Crawford D, Acuña J, Sherman S (2001) FMR1 and the fragile X syndrome: human genome epidemiology review. Genet Med 3:359-371.

Dahlhaus R (2018) Of men and mice: modeling the fragile X syndrome. Front Mol Neurosci 11:41.

Dalland T, Døving K (1981) Reaction to olfactory stimuli in odor-exposed rats. Behav Neural Biol 32:79-88.

Darnell J, Jensen K, Jin P, Brown V, Warren S, Darnell R (2001) Fragile X mental retardation protein targets $\mathrm{G}$ quartet mRNAs important for neuronal function. Cell 107:489-499.

Darnell J, Van Driesche S, Zhang C, Hung K, Mele A, Fraser C, Stone E, Chen C, Fak J, Chi S, Licatalosi D, Richter J, Darnell R (2011) FMRP stalls ribosomal translocation on mRNAs linked to synaptic function and autism. Cell 146:247-261.

Das A, Chiang A, Davla S, Priya R, Reichert H, VijayRaghavan K, Rodrigues V (2010) Identification and analysis of a glutamatergic local interneuron lineage in the adult Drosophila olfactory system. Neural Syst Circuits 1:4.

Das S, Sadanandappa M, Dervan A, Larkin A, Lee J, Sudhakaran I, Priya R, Heidari R, Holohan E, Pimentel A, Gandhi A, Ito K, Sanyal S, Wang J, Rodrigues V, Ramaswami M (2011) Plasticity of local GABAergic interneurons drives olfactory habituation. Proc Natl Acad Sci USA 108:E646E654.

Devaud J, Acebes A, Ramaswami M, Ferrús A (2003) Structural and functional changes in the olfactory pathway of adult Drosophila take place at a critical age. J Neurobiol 56:13-23.

Devaud J, Clouet-Redt C, Bockaert J, Grau Y, Parmentier M (2008) Widespread brain distribution of the Drosophila metabotropic glutamate receptor. Neuroreport 19:367-371.

Doll C, Broadie K (2015) Activity-dependent FMRP requirements in development of the neural circuitry of learning and memory. Development 142:1346-1356.

Doll C, Broadie K (2016) Neuron class-specific requirements for fragile $\mathrm{X}$ mental retardation protein in critical period development of calcium signaling in learning and memory circuitry. Neurobiol Dis 89:76-87.

Doll C, Vita D, Broadie K (2017) Fragile X mental retardation protein requirements in activity-dependent critical period neural circuit refinement. Curr Biol 27:2318-2330.

Dölen G, Osterweil E, Rao B, Smith G, Auerbach B, Chattarji S, Bear M (2007) Correction of fragile X syndrome in mice. Neuron 56:955962.

Domanski A, Booker S, Wyllie D, Isaac J, Kind P (2019) Cellular and synaptic phenotypes lead to disrupted information processing in Fmr1-KO mouse layer 4 barrel cortex. Nat Commun 10:4814.

Fishilevich E, Vosshall L (2005) Genetic and functional subdivision of the Drosophila antennal lobe. Curr Biol 15:1548-1553. 
Flockhart I, Booker M, Kiger A, Boutros M, Armknecht S, Ramadan N, Richardson K, Xu A, Perrimon N, Mathey-Prevot B (2006) FlyRNAi: the Drosophila RNAi screening center database. Nucleic Acids Res 34:D489D494.

Franco L, Okray Z, Linneweber G, Hassan B, Yaksi E (2017) Reduced lateral inhibition impairs olfactory computations and behaviors in a Drosophila model of fragile X syndrome. Curr Biol 27:1111-1123.

Friedman S, Dani N, Rushton E, Broadie K (2013) Fragile X mental retardation protein regulates trans-synaptic signaling in Drosophila. Dis Model Mech 6:1400-1413.

Geramita M, Urban N (2016) Postnatal odor exposure increases the strength of interglomerular lateral inhibition onto olfactory bulb tufted cells. J Neurosci 36:12321-12327.

Gerlach G, Tietje K, Biechl D, Namekawa I, Schalm G, Sulmann A (2019) Behavioural and neuronal basis of olfactory imprinting and kin recognition in larval fish. J Exp Biol 222:jeb189746.

Goel A, Cantu D, Guilfoyle J, Chaudhari G, Newadkar A, Todisco B, Alba D, Kourdougli N, Schmitt L, Pedapati E, Erickson C, Portera-Cailliau C (2018) Impaired perceptual learning in a mouse model of Fragile X syndrome is mediated by parvalbumin neuron dysfunction and is reversible. Nat Neurosci 21:1404-1411.

Golovin R, Vest J, Vita D, Broadie K (2019) Activity-dependent remodeling of Drosophila olfactory sensory neuron brain innervation during an early-life critical period. J Neurosci 39:2995-3012.

Gonçalves J, Anstey J, Golshani P, Portera-Cailliau C (2013) Circuit level defects in the developing neocortex of fragile $\mathrm{X}$ mice. Nat Neurosci 16:903-909.

Greenblatt E, Spradling A (2018) Fragile X mental retardation 1 gene enhances the translation of large autism-related proteins. Science 361:709-712.

Greenhill S, Juczewski K, Haan A, Seaton G, Fox K, Hardingham N (2015) Adult cortical plasticity depends on an early postnatal critical period. Science 349:424-427.

Harlow E, Till S, Russell T, Wijetunge L, Kind P, Contractor A (2010) Critical period plasticity is disrupted in the barrel cortex of FMR1 knockout mice. Neuron 65:385-398.

Hayashi S, Ito K, Sado Y, Taniguchi M, Akimoto A, Takeuchi H, Aigaki T, Matsuzaki F, Nakagoshi H, Tanimura T, Ueda R, Uemura T, Yoshihara M, Goto S (2002) GETDB, a database compiling expression patterns and molecular locations of a collection of gal4 enhancer traps. Genesis 34:58-61.

He Q, Nomura T, Xu J, Contractor A (2014) The developmental switch in GABA polarity is delayed in fragile X mice. J Neurosci 34:446-450.

Hensch T (2005) Critical period plasticity in local cortical circuits. Nat Rev Neurosci 6:877-888.

Hersh J, Saul R; Committee on Genetics (2011) Health supervision for children with fragile X syndrome. Pediatrics 127:994-1006.

Huang J, Zhang W, Qiao W, Hu A, Wang Z (2010) Functional connectivity and selective odor responses of excitatory local interneurons in Drosophila antennal lobe. Neuron 67:1021-1033.

Hubel D, Wiesel T (1970) The period of susceptibility to the physiological effects of unilateral eye closure in kittens. J Physiol 206:419436.

Inoue S, Shimoda M, Nishinokubi I, Siomi M, Okamura M, Nakamura A, Kobayashi S, Ishida N, Siomi H (2002) A role for the Drosophila fragile X-related gene in circadian output. Curr Biol 12:13311335.

Jackson F, Newby L, Kulkarni S (1990) Drosophila GABAergic systems: sequence and expression of glutamic acid decarboxylase. J Neurochem 54:1068-1078.

Jefferis G, Marin E, Watts R, Luo L (2002) Development of neuronal connectivity in Drosophila antennal lobes and mushroom bodies. Curr Opin Neurobiol 12:80-86.

Khandjian EW, Fortin A, Thibodeau A, Tremblay S, Côté F, Devys D, Mandel JL, Rousseau F (1995) A heterogeneous set of FMR1 proteins is widely distributed in mouse tissues and is modulated in cell culture. Hum Mol Genet 4:783-789.

Klapoetke N, Murata Y, Kim S, Pulver S, Birdsey-Benson A, Cho Y, Morimoto T, Chuong A, Carpenter E, Tian Z, Wang J, Xie Y, Yan Z, Zhang Y, Chow B, Surek B, Melkonian M, Jayaraman V, ConstantinePaton M, Wong G, et al. (2014) Independent optical excitation of distinct neural populations. Nat Methods 11:338-346.
Krishnan K, Wang B, Lu J, Wang L, Maffei A, Cang J, Huang Z (2015) $\mathrm{MeCP} 2$ regulates the timing of critical period plasticity that shapes functional connectivity in primary visual cortex. Proc Natl Acad Sci USA 112: E4782-E4791.

Larsson M, Domingos A, Jones W, Chiappe M, Amrein H, Vosshall L (2004) Or83b encodes a broadly expressed odorant receptor essential for Drosophila olfaction. Neuron 43:703-714.

Lee T, Luo L (1999) Mosaic analysis with a repressible cell marker for studies of gene function in neuronal morphogenesis. Neuron 22:451-461.

Liu A, Urban N (2017) Prenatal and early postnatal odorant exposure heightens odor-evoked mitral cell responses in the mouse olfactory bulb. eNeuro 4:ENEURO.0129-17.2017.

Liu W, Wilson R (2013) Glutamate is an inhibitory neurotransmitter in the Drosophila olfactory system. Proc Natl Acad Sci USA 110:10294-10299.

Liu X, Krause W, Davis R (2007) GABAA receptor RDL inhibits Drosophila olfactory associative learning. Neuron 56:1090-1102.

Lovelace J, Rais M, Palacios A, Shuai X, Bishay S, Popa O, Pirbhoy P, Binder D, Nelson D, Ethell I, Razak K (2020) Deletion of Fmr1 from forebrain excitatory neurons triggers abnormal cellular, EEG, and behavioral phenotypes in the auditory cortex of a mouse model of fragile $\mathrm{X}$ syndrome. Cereb Cortex 30:969-988.

Marin E, Jefferis G, Komiyama T, Zhu H, Luo L (2002) Representation of the glomerular olfactory map in the Drosophila brain. Cell 109:243-255.

Meredith R (2015) Sensitive and critical periods during neurotypical and aberrant neurodevelopment: a framework for neurodevelopmental disorders. Neurosci Biobehav Rev 50:180-188.

Mohamed A, Retzke T, Chakraborty S, Fabian B, Hansson B, Knaden M, Sachse S (2019) Odor mixtures of opposing valence unveil interglomerular crosstalk in the Drosophila antennal lobe. Nat Commun 10:1201.

Mohammad F, Stewart J, Ott S, Chlebikova K, Chua J, Koh T, Ho J, ClaridgeChang A (2017) Optogenetic inhibition of behavior with anion channelrhodopsins. Nat Methods 14:271-274.

Münch D, Galizia C (2016) DoOR 2.0-comprehensive mapping of Drosophila melanogaster odorant responses. Sci Rep 6:21841.

Nagarkar-Jaiswal S, DeLuca S, Lee P, Lin W, Pan H, Zuo Z, Lv J, Spradling A, Bellen $\mathrm{H}$ (2015) A genetic toolkit for tagging intronic MiMIC containing genes. Elife 4:e08469.

Okada R, Awasaki T, Ito K (2009) Gamma-aminobutyric acid (GABA)-mediated neural connections in the Drosophila antennal lobe. J Comp Neurol 514:74-91.

Olsen S, Wilson R (2008) Lateral presynaptic inhibition mediates gain control in an olfactory circuit. Nature 452:956-960.

Olsen S, Bhandawat V, Wilson R (2010) Divisive normalization in olfactory population codes. Neuron 66:287-299.

Roberts J, McCary L, Shinkareva S, Bailey D (2016) Infant development in fragile X syndrome: cross-syndrome comparisons. J Autism Dev Disord 46:2088-2099.

Rorth P (1996) A modular misexpression screen in Drosophila detecting tissuespecific phenotypes. Proc Natl Acad Sci USA 93:12418-12422.

Sachse S, Rueckert E, Keller A, Okada R, Tanaka N, Ito K, Vosshall L (2007) Activity-dependent plasticity in an olfactory circuit. Neuron 56:838-850

Sawicka K, Hale C, Park C, Fak J, Gresack J, Driesche S, Kang J, Darnell J, Darnell R (2019) FMRP has a cell-type-specific role in CA1 pyramidal neurons to regulate autism-related transcripts and circadian memory. Elife 8:e46919.

Schindelin J, Arganda-Carreras I, Frise E, Kaynig V, Longair M, Pietzsch T, Preibisch S, Rueden C, Saalfeld S, Schmid B, Tinevez J, White D, Hartenstein V, Eliceiri K, Tomancak P, Cardona A (2012) Fiji: an open-source platform for biological-image analysis. Nat Methods 9:676-682.

Sears J, Choi W, Broadie K (2019) Fragile X mental retardation protein positively regulates PKA anchor rugose and PKA activity to control actin assembly in learning/memory circuitry. Neurobiol Dis 127:53-64.

Semmelhack J, Wang J (2009) Select Drosophila glomeruli mediate innate olfactory attraction and aversion. Nature 459:218-223.

Stephan D, Sánchez-Soriano N, Loschek L, Gerhards R, Gutmann S, Storchova Z, Prokop A, Kadow I (2012) Drosophila psidin regulates 
olfactory neuron number and axon targeting through two distinct molecular mechanisms. J Neurosci 32:16080-16094.

Stocker R, Heimbeck G, Gendre N, Belle J (1997) Neuroblast ablation in Drosophila P[GAL4] lines reveals origins of olfactory interneurons. J Neurobiol 32:443-456.

Sudhakaran I, Hillebrand J, Dervan A, Das S, Holohan E, Hülsmeier J, Sarov M, Parker R, VijayRaghavan K, Ramaswami M (2014) FMRP and Ataxin-2 function together in long-term olfactory habituation and neuronal translational control. Proc Natl Acad Sci USA 111: E99-E108.

Sweeney L, Couto A, Chou Y, Berdnik D, Dickson B, Luo L, Komiyama T (2007) Temporal target restriction of olfactory receptor neurons by semaphorin-1a/plexinA-mediated axon-axon interactions. Neuron 53:185-200.

Sweeney S, Broadie K, Keane J, Niemann H, O'Kane C (1995) Targeted expression of tetanus toxin light chain in Drosophila specifically eliminates synaptic transmission and causes behavioral defects. Neuron 14:341-351.

Tessier C, Broadie K (2009) Activity-dependent modulation of neural circuit synaptic connectivity. Front Mol Neurosci 2:8.

Wang J, Beck E, McCabe B (2012) A modular toolset for recombination transgenesis and neurogenetic analysis of Drosophila. PLoS One 7:e42102.
Wilson R (2013) Early olfactory processing in Drosophila: mechanisms and principles. Annu Rev Neurosci 36:217-241.

Wodarz A, Hinz U, Engelbert M, Knust E (1995) Expression of crumbs confers apical character on plasma membrane domains of ectodermal epithelia of Drosophila. Cell 82:67-76.

Xia S, Miyashita T, Fu T, Lin W, Wu C, Pyzocha L, Lin I, Saitoe M, Tully T, Chiang A (2005) NMDA receptors mediate olfactory learning and memory in Drosophila. Curr Biol 15:603-615.

Yu F, Schuldiner O (2014) Axon and dendrite pruning in Drosophila. Curr Opin Neurobiol 27:192-198.

Zalfa F, Giorgi M, Primerano B, Moro A, Penta A, Reis S, Oostra B, Bagni C (2003) The Fragile X syndrome protein FMRP associates with BC1 RNA and regulates the translation of specific mRNAs at synapses. Cell 112:317-327.

Zars T, Fischer M, Schulz R, Heisenberg M (2000) Localization of a short-term memory in Drosophila. Science 288:672-675.

Zhang Y, Bailey A, Matthies H, Renden R, Smith M, Speese S, Rubin G, Broadie K (2001) Drosophila fragile X-related gene regulates the MAP1B homolog Futsch to control synaptic structure and function. Cell 107:591-603. 